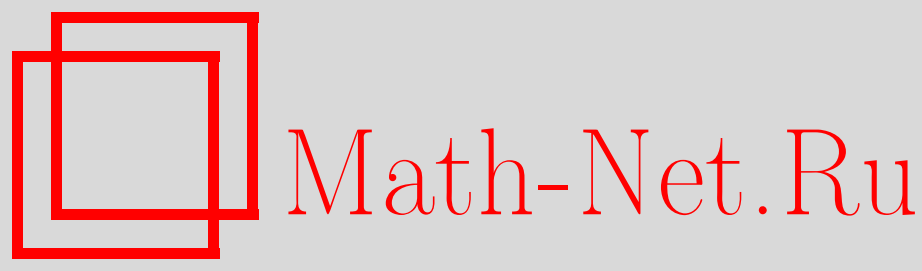

Е. Г. Гельфер, А. М. Федотов, В. Д. Мур, Н. Б. Нарожный, Бустовы моды массивного фермионного поля и задача Унру, ТМФ, 2015, том 182, номер 3, 405-434

DOI: https://doi.org/10.4213/tmf8690

Использование Общероссийского математического портала Math-Net.Ru подразумевает, что вы прочитали и согласны с пользовательским соглашением http://www . mathnet.ru/rus/agreement

Параметры загрузки:

IP: 54.84 .234 .179

26 апреля 2023 г., 14:38:41

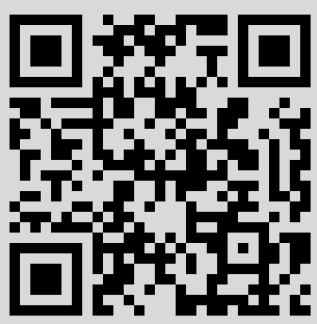




\title{
ФИЗИКА
}

Том 182, № 3

март, 2015

(C) 2015 г.

\author{
Е. Г. Гельфер*, А. М. Федотов*, \\ В. Д. Мур*, Н. Б. Нарожный*

\section{БУСТОВЫ МОДЫ МАССИВНОГО ФЕРМИОННОГО ПОЛЯ И ЗАДАЧА УНРУ}

Показано, что функция Вайтмана свободного квантованного поля порождает любой полный набор решений релятивистских волновых уравнений. С использованием этого подхода построен полный набор решений двумерного уравнения Дирака, состоящий из собственных функций генератора лоренцевых поворотов (оператора буста). Показано, что на поверхности светового конуса бустовы моды фермионного поля содержат дельта-функции Гельфанда комплексного аргумента. Вследствие такой сингулярности исключение даже одной единственной моды с произвольным значением бустового квантового числа делает набор бустовых мод неполным. Это приводит к тому, что схема квантования Унру неприменима к массивному фермионному полю в двумерном пространстве Минковского. Следовательно, в полном соответствии с бозонным случаем, процедура квантования Унру для фермионного поля не может быть использована для обоснования эффекта Унру.

Ключевые слова: бустова симметрия, фермионное поле, функция Вайтмана, нулевая мода.

DOI: $10.4213 / \operatorname{tmf} 8690$

\section{1. ВВЕДЕНИЕ}

В данной работе мы рассматриваем задачу Унру для массивного фермионного поля в двумерном пространстве Минковского (ПМ). Случай массивного скалярного поля был впервые рассмотрен Унру в его знаменитой работе [1], основной результат которой состоит в следующем: в ПМ существует однопараметрическое семейство вакуумных состояний, унитарно неэквивалентных стандартному вакуумному состоянию квантованного поля. Согласно Унру это утверждение эквивалентно тому, что детектор, движущийся в пустом ПМ с постоянным ускорением $g$, будет вести себя так же, как если бы находился в тепловой бане при универсальной температуре Девиса-Унру $T_{\mathrm{DU}}=g \hbar / 2 \pi c[1],[2]$. Самой важной особенностью этого утверждения является универсальность отклика, т. е. его независимость от устройства детектора, природы взаимодействия с квантованным полем и ускоряющей силы.

*Национальный исследовательский ядерный университет "МИФИ", Москва, Россия. E-mail: egelfer@gmail.com 
В ряде работ [3]-[6] (см. также [7]-[10]) было показано, что статья Унру [1] содержит нетривиальную математическую ошибку, которая делает выводы статьи неверными. Тем не менее многие авторы не согласны с этим мнением. Более того, в последнее время появилось много предложений по проверке эффекта Унру в экспериментах по взаимодействию ультрарелятивистских частиц со сверхсильными лазерными полями [11]-[13]. Также обсуждалась возможность наблюдения основанных на эффекте Унру перепутанных состояний как бозонов, так и фермионов [14]-[18]. В то же время другие авторы [19] считают, что такие предложения основаны на недоразумении. В связи с этим строгое исследование задачи Унру остается насущной задачей современной физики. Особенно это относится ко все еще слабо изученному фермионному случаю, потому что свойства фермионных бустовых мод, которые являются базисом для квантования Унру, существенно отличаются от свойств их бозонных аналогов.

Как уже было отмечено выше, существуют два аспекта задачи Унру: аспект квантовой теории поля (КТП) и детекторный аспект. Детекторный аспект обсуждался многими авторами, при этом выводы различных работ противоречивы. В одних работах были продемонстрированы модели детекторов, обладающих тепловым откликом Девиса-Унру (см., например, [1], недавнюю статью [20] и обзоры [21], [22]), в других [23]-[26] были предложены модели без теплового отклика. В любом случае соверешенно очевидно, что невозможно доказать универсальность эффекта Унру, исследуя различные модели детекторов. Поэтому в данной работе мы сосредоточимся только на КТП-аспекте задачи Унру.

Хорошо известно, что процедура квантования в КТП предполагает разложение оператора поля в ряд по полному набору мод, являющихся решениями соответствующего классического полевого уравнения (Дирака или Клейна-Фока-Гордона (КФГ)). Поэтому поиск точных решений этих уравнения является одной из основных задач КТП в присутствии классического поля.

Один из самых мощных инструментов решения уравнений в частных производных основан на использовании свойств симметрии физической системы, которую это уравнение описывает. По теореме Нётер [27] каждой непрерывной симметрии действия физической системы соответствует закон сохранения ${ }^{1)}$. При этом всегда можно выбрать набор переменных таким образом, чтобы сдвиг по одной из них соответствовал преобразованию симметрии. Поскольку генератор преобразования симметрии коммутирует с оператором дифференциального уравнения, соответствующая переменная может быть отделена. Если уравнение имеет достаточное количество симметрий и их генераторы коммутируют между собой, то задача о нахождении решений уравнения, вообще говоря, может быть сведена к решению обыкновенного дифференциального уравнения второго порядка. Полученные решения нумеруются собственными значениями генераторов симметрий, и их набор полон.

Все самые важные и широко используемые решения релятивистских квантовых уравнений в присутствии внешнего классического поля были получены с помощью этого метода. В их числе кулоновское поле (см., например, [29]), постоянное магнитное [29] и электрическое [30], [31] поля, поле плоской электромагнитной волны [32] и некоторые другие поля более сложной конфигурации [31], [33]. Во всех этих случаях решения были собственными функциями линейных комбинаций генераторов

\footnotetext{
1) Точнее, преобразований, оставляющих форм-инвариантные уравнения движения [28].
} 
трансляций в пространстве и времени или пространственных поворотов и нумеровались значениями компонент 4-вектора энергии-импульса или момента импульса соответственно. Однако группа Пуанкаре изометрий ПМ включает в себя также лоренцевы повороты (бусты). Бустова симметрия почти никогда не использовалась для квантования поля. Причиной этого является то, что оператор буста не коммутирует с гамильтонианом, и поэтому бустовое квантовое число и энергия не могут входить в полный набор наблюдаемых одновременно. Тем не менее бустовы моды могут быть очень полезны, особенно для КТП в искривленном пространстве или если симметрия ПМ по отношению к трансляциям во времени и/или пространстве нарушена присутствием внешнего классического поля. В этих случаях бустова симметрия может оказаться единственной симметрией квантованного поля. В качестве примеров можно привести дилатонную гравитацию в двух измерениях [34], а также геометрию Шварцшильда [35]. По этой причине анализ свойств бустовых мод является важной задачей.

Впервые бустовы моды свободного массивного скалярного поля обсуждались в статье Унру [1], однако в явном виде они там приведены не были. Затем некоторые свойства скалярных бустовых мод были изучены в работах [6], [23], [36]. В частности, замечательные свойства бустовых мод в двумерном ПМ были установлены в работе [6], где было показано, что, во-первых, нулевая бустова мода свободного массивного скалярного поля совпадает с точностью до постоянного множителя с положительно-частотной функцией Вайтмана и, во-вторых, бустовы моды как функции бустового квантового числа обладают сингулярностью типа дельта-функции на поверхности светового конуса.

Автор работы [1] не обратил внимания на эту сингулярность и при построении нового набора мод Унру, являющихся особыми комбинациями бустовых мод, исключил сингулярную нулевую моду из полного набора. В результате набор мод Унру оказался неполным и потому не мог служить базисом для квантования свободного поля в ПМ. Ту же ошибку совершили и авторы [37], [38].

Фуллинг и Унру [39] сделали попытку реабилитировать эффект Унру: они продекларировали, что сглаживание поля в духе теории обобщенных функций Шварца обратит в нуль вклад сингулярной бустовой моды, сделав квантование Унру в ПМ корректным. Тем не менее очевидно, что квантованное поле не может существовать в отсутствие функции Вайтмана. Это утверждение было подтверждено в ответе [40] на комментарий Фуллинга и Унру, где было строго доказано, что сглаживание не исправляет ситуацию. Детали можно найти в работах [6], [8], а также в разделе 2 и в приложении А настоящей статьи.

Еще интереснее оказались свойства бустовых мод в случае свободного безмассового фермионного поля. Как было показано в [41], бустовы моды двумерных безмассовых фермионов на световом конусе выражаются через дельта-функцию комплексного аргумента. Бустовы моды массивного фермионного поля впервые были рассмотрены в работах [42], [43] (см. также [44]). Тем не менее результаты [42]-[44] содержат некоторые неточности, которые мы обсудим в разделе 3 настоящей статьи.

В этой работе мы рассматриваем бустовы моды свободного массивного фермионного поля. Для того чтобы их построить, мы используем функции Вайтмана. Положительно- и отрицательно-частотные функции Вайтмана свободных полей полностью определяются (с точностью до постоянного множителя) инвариантностью 
теории относительно преобразований Лоренца и трансляций [45]. И наоборот, если известна функция Вайтмана, эти симметрии могут быть использованы для построения любого полного набора положительно- и отрицательно-частотных решений уравнений КФГ и Дирака. Фактически это прямое следствие теоремы реконструкции Вайтмана [46], [47].

Поскольку лоренцев поворот выделяет двумерную плоскость в ПМ, мы обсудим специфические свойства бустовых мод на примере двумерных уравнений КФГ и Дирака. В разделе 2 мы реализуем сформулированный выше подход на примере однокомпонентного массивного нейтрального поля. Следует отметить, что, в отличие от случая плосковолновых мод, аналитические свойства бустовых мод разительно меняются при переходе к многокомпонентным полям, которые рассмотрены в разделе 3. Обсуждение результатов и выводы представлены в разделе 4. Некоторые технические детали обсуждаются в приложении А.

\section{2. БОзОНЫ}

Рассмотрим случай свободного нейтрального массивного скалярного поля в двумерном ПМ. Начнем с двухточечной функции Вайтмана [46], которая в теории свободного поля совпадает с положительно-частотной частью коммутатора двух операторов поля (функции Паули-Йордана в четырехмерной скалярной теории). Положительно-частотная функция Вайтмана $\Delta^{(+)}(x), x=(t, z)$, для массивного скалярного поля удовлетворяет уравнению КФГ ${ }^{2)}$

$$
\mathcal{K} \Delta^{(+)}(x)=0, \quad \mathcal{K}=\partial_{t}^{2}-\partial_{z}^{2}+m^{2},
$$

содержит только положительные частоты и инвариантна относительно лоренцевых поворотов (бустов)

$$
\mathcal{B}_{\mathcal{K}} \Delta^{(+)}(x)=0, \quad \mathcal{B}_{\mathcal{K}}=i\left(z \partial_{t}+t \partial_{z}\right)
$$

Этими условиями $\Delta^{(+)}(x)$ определяется с точностью до постоянного множителя. Действительно, любое положительно-частотное решение уравнения КФГ можно представить в виде

$$
\Phi^{(+)}(x)=\int d^{2} p \phi(p) \delta\left(p^{2}-m^{2}\right) \theta\left(p^{0}\right) e^{-i p x},
$$

где $\phi(p)$ - некоторая функция 2-вектора $p=\left(p^{0}, p^{1}\right), \theta\left(p^{0}\right)$ - ступенчатая функция Хевисайда. После замены переменных

$$
p^{0}=\mu \operatorname{ch} q, \quad p^{1}=\mu \operatorname{sh} q,
$$

где $q$ - быстрота, и интегрирования по $\mu$ получаем уравнение

$$
\Phi^{(+)}(x)=\frac{1}{2} \int_{-\infty}^{\infty} d q \phi(m \operatorname{ch} q, m \operatorname{sh} q) e^{-i m(t \operatorname{ch} q-z \operatorname{sh} q)} .
$$

\footnotetext{
2) В данной статье мы используем естественные единицы $\hbar=c=1$.
} 
Легко видеть, что условие (2) выполняется только для $\phi(q)=$ const. Выбирая $\phi(q)=$ $i / 2 \pi$, приходим к стандартному представлению для $\Delta^{(+)}(x)$ (ср., например, [6], [27]):

$$
\Delta^{(+)}(x)=\frac{i}{4 \pi} \int_{-\infty}^{\infty} d q e^{-i m(t \operatorname{ch} q-z \operatorname{sh} q)} .
$$

При этом предполагается, что в (5) к $t$ имеется бесконечно малая мнимая отрицательная добавка [6]. Другое представление для $\Delta^{(+)}(x)$ имеет вид (см., например, [48])

$$
\Delta^{(+)}(x)=\frac{i}{4 \pi} \int_{-\infty}^{\infty} \frac{d p}{\varepsilon_{p}} e^{-i \varepsilon_{p} t+i p z}, \quad \varepsilon_{p}=\sqrt{p^{2}+m^{2}},
$$

здесь и далее будем опускать индекс пространственной компоненты 2-вектора $p$.

По теореме реконструкции Вайтмана [46] двухточечная функция Вайтмана однозначно определяет квантовую теорию свободного поля. В частности, она позволяет построить любой ортонормированный полный набор решений полевого уравнения. В самом деле, из трансляционной инвариантности теории следует, что функция Вайтмана с произвольно сдвинутым аргументом

$$
\Delta^{(+)}(x-u), \quad u=\left\{u^{0}, u^{1}\right\},
$$

удовлетворяет уравнению КФГ. Функции (7) образуют переполненный набор мод, поскольку они нумеруются двумя независимыми параметрами $u^{0}, u^{1}$. В любом случае, любой полный набор положительно-частотных решений $F_{a}(x)$,

$$
\int d a F_{a}\left(x^{\prime}\right) F_{a}^{*}\left(x^{\prime \prime}\right)=-i \Delta^{(+)}\left(x^{\prime}-x^{\prime \prime}\right),
$$

ортонормированных согласно

$$
\left(F_{a}, F_{a^{\prime}}\right) \equiv i \int_{-\infty}^{\infty} F_{a}^{*}(x) \stackrel{\leftrightarrow}{\partial}_{t} F_{a^{\prime}}(x) d z=\delta\left(a-a^{\prime}\right)
$$

можно представить в виде

$$
F_{a}(x)=\int d^{2} u f_{a}(u) \Delta^{(+)}(x-u) .
$$

Однако при этом, поскольку набор решений (7) переполнен, коэффициентные функции $f_{a}(u)$ не могут быть определены однозначно. Для того чтобы сделать выбор $f_{a}(u)$ однозначным, следует наложить некоторое ограничение на $u$, которое может быть выбрано из соображений симметрии.

Для начала проиллюстрируем эту процедуру для тривиального случая плоских волн $\Theta_{p}(x)$, которые являются собственными функциями генератора пространственных трансляций

$$
-i \partial_{z} \Theta_{p}(x)=p \Theta_{p}(x) .
$$

В этом случае удобно ограничить семейство $\Delta^{(+)}(x-u)$ только пространственно сдвинутыми друг относительно друга функциями. Это означает, что коэффициентные функции $f_{p}(u)$ имеют вид $f_{p}(u)=\delta\left(u^{0}\right) \vartheta_{p}\left(u^{1}\right)$, и поэтому

$$
\Theta_{p}(x)=\int_{-\infty}^{\infty} d u^{1} \vartheta_{p}\left(u^{1}\right) \Delta^{(+)}\left(t, z-u^{1}\right) .
$$


Подставим это разложение в (11). Учитывая, что функция Вайтмана $\Delta^{(+)}(t, z)$ экспоненциально убывает при $|z| \rightarrow \infty$, можно проинтегрировать левую часть полученного соотношения по частям, что приводит к следующему уравнению для $\vartheta_{p}\left(u^{1}\right)$ :

$$
-i \vartheta_{p}^{\prime}\left(u^{1}\right)=p \vartheta_{p}\left(u^{1}\right),
$$

откуда $\vartheta_{p}\left(u^{1}\right)=\mathrm{const} \cdot e^{i p u^{1}}$. Подставляя теперь $\vartheta_{p}\left(u^{1}\right)$ в таком виде в $(12)$ и используя (6), после интегрирования по $u^{1}$ окончательно получаем

$$
\Theta_{p}(x)=\frac{1}{\sqrt{4 \pi \varepsilon_{p}}} e^{-i \varepsilon_{p} t+i p z} .
$$

Здесь нормировочная константа определена из условия (9).

Функции $\Theta_{p}(x)$ удовлетворяют соотношению

$$
\int_{-\infty}^{\infty} d p \Theta_{p}\left(x^{\prime}\right) \Theta_{p}^{*}\left(x^{\prime \prime}\right)=-i \Delta^{(+)}\left(x^{\prime}-x^{\prime \prime}\right),
$$

и поэтому набор (13) полон.

Нас интересует набор бустовых мод $\Psi_{\kappa}^{(+)}(x)$, являющихся собственными функциями оператора буста $\mathcal{B}_{\mathcal{K}}$ :

$$
\mathcal{B}_{\mathcal{K}} \Psi_{\kappa}^{(+)}(x)=\kappa \Psi_{\kappa}^{(+)}(x) .
$$

Прежде всего отметим, что функция Вайтмана $\Delta^{(+)}(x)$ является нулевой бустовой модой $\Psi_{0}^{(+)}(x)$ этого набора, что следует из уравнений $(15)$ и $(2)$.

Чтобы получить бустовы моды, ограничим $u$ в (10) одной из орбит собственной ортохронной группы Лоренца,

$$
u^{2}=u^{0^{2}}-u^{1^{2}}= \pm v^{2}, \quad v=\text { const }
$$

так что $f(u) \sim \delta\left(u^{2} \mp v^{2}\right)$. Выбрав для определенности верхний знак в $(16)$, положим

$$
u \equiv u_{q}=(v \operatorname{ch} q, v \operatorname{sh} q)
$$

и перепишем уравнение (10) в виде

$$
\Psi_{\kappa}^{(+)}(x)=\int_{-\infty}^{\infty} d q \zeta_{\kappa}(q) \Delta^{(+)}\left(x-u_{q}\right) .
$$

Подставляя (18) в (15) и используя соотношение

$$
\mathcal{B}_{\mathcal{K}} \Delta^{(+)}\left(x-u_{q}\right)=-i \frac{\partial}{\partial q} \Delta^{(+)}\left(x-u_{q}\right),
$$

которое можно получить непосредственно с помощью представления (5), приходим к уравнению для $\zeta_{\kappa}(q)$ :

$$
i \frac{\partial \zeta_{\kappa}(q)}{\partial q}=\kappa \zeta_{\kappa}(q)
$$

Отсюда $\zeta_{\kappa}(q)=\mathrm{const} \cdot e^{-i \kappa q}$, и, наконец, из (18) с учетом (9) получаем следующее представление для положительно-частотных бустовых мод:

$$
\Psi_{\kappa}^{(+)}(x)=\frac{1}{2^{3 / 2} \pi} \int_{-\infty}^{\infty} d q e^{-i m(t \operatorname{ch} q-z \operatorname{sh} q)-i \kappa q},
$$


которое было ранее получено в [6], [23], [36] другими способами. Отрицательно-частотные моды $\Psi_{\kappa}^{(-)}(x)$ определяются [6] как

$$
\Psi_{\kappa}^{(-)}(x)=\Psi_{-\kappa}^{(+)^{*}}(x) .
$$

Бустовы моды (20), (21) являются обобщенными функциями по отношению как к $x=(t, z)$, так и к спектральному параметру $\kappa$. Они определены на классе гладких достаточно быстро убывающих функций.

Моды (20), (21) составляют полный набор, поскольку удовлетворяют условию

$$
\int_{-\infty}^{\infty} d \kappa \Psi_{\kappa}^{( \pm)}\left(x^{\prime}\right) \Psi_{\kappa}^{( \pm)^{*}}\left(x^{\prime \prime}\right)=\mp i \Delta^{( \pm)}\left(x^{\prime}-x^{\prime \prime}\right),
$$

поэтому они могут быть использованы в качестве базиса для квантования нейтрального скалярного поля (см. [6]). Поскольку знак энергии частицы является лоренцевым инвариантом, вакуумные состояния в бустовой и плосковолновой схемах квантования совпадают. Следовательно, эти две схемы квантования унитарно эквивалентны (см. [6]).

Самым примечательным свойством бустовых мод является их поведение на световом конусе. Из (20), (21) несложно заметить, что в вершине светового конуса функция $\Psi_{\kappa}^{( \pm)}(x)$ имеет особенность вида дельта-функции Дирака:

$$
\Psi_{\kappa}^{( \pm)}(0)=\frac{1}{\sqrt{2}} \delta(\kappa)
$$

Как показано в $[6], \Psi_{\kappa}^{( \pm)}(x)$ имеет особенность вида дельта-функции также и на образующих светового конуса $x_{ \pm} \equiv t \pm z=0$. Поэтому вклад единственной спектральной точки $\kappa=0$ в физические величины может оказаться конечным. Мы проиллюстрируем это на примере функции Вайтмана.

Вследствие трансляционной инвариантности можно переписать (22) в виде

$$
\Delta^{(+)}\left(x^{\prime}-x^{\prime \prime}\right)=i \int_{-\infty}^{\infty} d \kappa \Psi_{\kappa}^{(+)}\left(x^{\prime}\right) \Psi_{\kappa}^{(+)^{*}}\left(x^{\prime \prime}\right)=i \int_{-\infty}^{\infty} d \kappa \Psi_{\kappa}^{(+)}\left(x^{\prime}-x^{\prime \prime}\right) \Psi_{\kappa}^{(+)^{*}}(0) .
$$

Тогда, используя (23), получим

$$
\Delta^{(+)}\left(x^{\prime}-x^{\prime \prime}\right)=\frac{i}{\sqrt{2}} \Psi_{0}^{(+)}\left(x^{\prime}-x^{\prime \prime}\right),
$$

т. е. интеграл по $\kappa$ в (24) полностью определяется ${ }^{3)}$ вкладом точки $\kappa=0$.

Этот результат означает, что точка $\kappa=0$ не может быть удалена из спектра, или, иными словами, интеграл по $\kappa$ в $(22),(24)$ не может быть заменен на свое главное значение:

$$
\int_{-\infty}^{\infty} d \kappa \cdots \neq \text { P.v. } \int_{-\infty}^{\infty} d \kappa \ldots
$$

3) Как уже было отмечено, выражение (25) прямо следует из выражений (2) и (15) с точностью до постоянного множителя. Поэтому (23) можно вывести из (25), (24) даже без использования представления (20). 
Таким образом, после исключения нулевой моды семейство бустовых мод уже не является полным набором в ПМ. Тем не менее в (26) равенство может быть восстановлено, если вырезать из ПМ световой конус (см. [6]). Это связано с тем, что все точки, в которых бустовы моды имеют особенность $\delta(\kappa)$, расположены именно на световом конусе.

Более того, функция Вайтмана (24) не может быть представлена в виде

$$
\widetilde{\Delta}^{(+)}\left(x^{\prime}-x^{\prime \prime}\right)=i \int_{0}^{\infty} d \kappa\left\{\Psi_{\kappa}^{(+)}\left(x^{\prime}\right) \Psi_{\kappa}^{(+)^{*}}\left(x^{\prime \prime}\right)+\Psi_{-\kappa}^{(+)}\left(x^{\prime}\right) \Psi_{-\kappa}^{(+)}\left(x^{\prime \prime}\right)^{*}\right\} .
$$

Если бы это было не так, то вследствие трансляционной инвариантности и свойства (23) бустовых мод функцию (27) можно было бы переписать в виде

$$
\widetilde{\Delta}^{(+)}\left(x^{\prime}-x^{\prime \prime}\right)=\frac{i}{\sqrt{2}} \int_{0}^{\infty} d \kappa\left\{\Psi_{\kappa}^{(+)}\left(x^{\prime}-x^{\prime \prime}\right)+\Psi_{-\kappa}^{(+)}\left(x^{\prime}-x^{\prime \prime}\right)\right\} \delta(\kappa) .
$$

Однако выражение (28), очевидно, бессмысленно. Действительно, обобщенная функция $\delta(\kappa)$ определена на функциях, непрерывных на интервале, содержащем точку $\kappa=0$. Поэтому уравнение (28) должно пониматься как

$$
\widetilde{\Delta}^{(+)}\left(x^{\prime}-x^{\prime \prime}\right)=\frac{i}{\sqrt{2}} \int_{-\infty}^{\infty} d \kappa\left\{\Psi_{\kappa}^{(+)}\left(x^{\prime}-x^{\prime \prime}\right)+\Psi_{-\kappa}^{(+)}\left(x^{\prime}-x^{\prime \prime}\right)\right\} \theta(\kappa) \delta(\kappa),
$$

где $\theta(\kappa)$ - ступенчатая функция Хевисайда. Однако произведение двух обобщенных функций $\theta(\kappa) \delta(\kappa)$ не определено.

Авторы [49] не согласны с этим утверждением. Обсуждая свое уравнение (2.129), которое в наших обозначениях совпадает с (27), они признают, что это выражение не определено, если обе точки $x^{\prime}, x^{\prime \prime}$ расположены на световом конусе. Однако они считают, что сглаживание обобщенной функции $\Delta^{(+)}\left(x^{\prime}-x^{\prime \prime}\right)$ с помощью функций $f\left(x^{\prime}\right)$ и $g\left(x^{\prime \prime}\right)$ с компактным носителем исправит ситуацию, так что сглаженная "функция Вайтмана" (см. формулу (2.131) в работе [49]) хорошо определена уже во всем ПМ. Однако наш анализ показывает, что это не так. В самом деле, если воспользоваться (27) в виде (28), то видно, что это выражение не определено при произволъных $x^{\prime}$, $x^{\prime \prime}$. Более того, сглаживание не может улучшить ситуацию, поскольку не влияет на дельта-функцию в (28). Правильное выражение для сглаженной функции Вайтмана может быть легко получено из (24). Оно имеет вид

$$
\begin{aligned}
\Delta^{(+)}(f, g) & =\int d^{2} x^{\prime} d^{2} x^{\prime \prime} f^{*}\left(x^{\prime}\right) g\left(x^{\prime \prime}\right) \Delta^{(+)}\left(x^{\prime}-x^{\prime \prime}\right)= \\
& =i \int_{-\infty}^{\infty} d \kappa \Psi_{\kappa}^{(+)}(f, g) \Psi_{\kappa}^{(+)^{*}}(0)=\frac{i}{\sqrt{2}} \Psi_{0}^{(+)}(f, g),
\end{aligned}
$$

где

$$
\Psi_{\kappa}^{(+)}(f, g)=\int d^{2} x^{\prime} d^{2} x^{\prime \prime} f^{*}\left(x^{\prime}\right) g\left(x^{\prime \prime}\right) \Psi_{\kappa}^{(+)}\left(x^{\prime}-x^{\prime \prime}\right)
$$

- сглаженная бустова мода. Видно, что снова вклад в интеграл (30) для сглаженной функции Вайтмана дает единственная спектральная точка $\kappa=0$ (подробнее см. приложение А). 
Отметим, что можно было бы попробовать использовать выражение (27) непосредственно, не применяя к нему свойство трансляционной инвариантности. В таком случае нужно сначала придать точный математический смысл выражению (27). А именно, следует записать его в виде

$$
\widetilde{\Delta}^{(+)}\left(x^{\prime}, x^{\prime \prime}\right)=i \lim _{\varepsilon \rightarrow 0} \int_{\varepsilon}^{\infty} d \kappa\left\{\Psi_{\kappa}^{(+)}\left(x^{\prime}\right) \Psi_{\kappa}^{(+)^{*}}\left(x^{\prime \prime}\right)+\Psi_{-\kappa}^{(+)}\left(x^{\prime}\right) \Psi_{-\kappa}^{(+)^{*}}\left(x^{\prime \prime}\right)\right\} .
$$

Однако легко видеть, что функция $\widetilde{\Delta}^{(+)}\left(x^{\prime}, x^{\prime \prime}\right)$ в $(32)$ трансляционно-неинвариантна и потому не может иметь ничего общего с функцией Вайтмана свободного поля в ПМ. Действительно, если бы она была трансляционно-инвариантна, можно было бы записать ее в виде

$$
\widetilde{\Delta}^{(+)}\left(x^{\prime}, x^{\prime \prime}\right)=\frac{i}{\sqrt{2}} \lim _{\varepsilon \rightarrow 0} \int_{\varepsilon}^{\infty} d \kappa\left\{\Psi_{\kappa}^{(+)}\left(x^{\prime}-x^{\prime \prime}\right)+\Psi_{-\kappa}^{(+)}\left(x^{\prime}-x^{\prime \prime}\right)\right\} \delta(\kappa),
$$

откуда следовало бы, что она равна нулю тождественно.

Сингулярное поведение бустовых мод в точке $\kappa=0$ можно понять в терминах классических траекторий свободных частиц с заданным значением бустового параметра

$$
\kappa=z \varepsilon_{p}-t p
$$

где $p=m \dot{z} / \sqrt{1-\dot{z}^{2}}-$ импульс, $\varepsilon_{p}=m / \sqrt{1-\dot{z}^{2}}-$ энергия частицы, $\dot{z}=d z / d t$. Перепишем (34) в виде

$$
z=\dot{z} t+\frac{\kappa}{m} \sqrt{1-\dot{z}^{2}} .
$$

Продифференцировав обе стороны уравнения (35) по времени, нетрудно найти его общее решение

$$
z=v t+\frac{\kappa}{m} \sqrt{1-v^{2}}, \quad v=\text { const }, \quad|v|<1,
$$

и особое решение

$$
z=\operatorname{sgn}(\kappa) \sqrt{t^{2}+\frac{\kappa^{2}}{m^{2}}} .
$$

При $\kappa \neq 0$ особое решение представлено двумя ветвями гиперболы (37). Ветвь с $\kappa>0$ расположена в правом секторе ПМ, а ветвь с $\kappa<0-$ в левом (см. рис. 1а, 1б). Свободные бустовы частицы с заданным значением $\kappa$ распространяются вдоль мировых линий, которые представляют собой прямые, касательные к соответствующим ветвям гиперболы (37). Следует отметить, что классические частицы с положительными значениями бустового параметра $\kappa$ не могут проникать в левый сектор, а частицы с $\kappa<0-$ в правый.

Мировые линии бустовых частиц с $\kappa=0$ имеют вид

$$
z=v t
$$

Все они пересекают вершину светового конуса $h_{0}$, и любая мировая линия, пересекающая $h_{0}$, принадлежит семейству траекторий с $\kappa=0$ (см. рис. 1в). Поэтому исключение из спектра точки $\kappa=0$ эквивалентно запрету частицам проходить через точку $h_{0}$, или, другими словами, выкалыванию этой точки из ПМ. В частности, 
$\kappa>0$

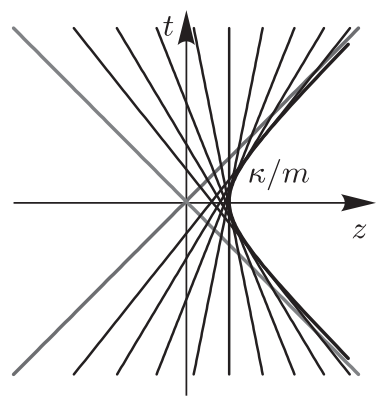

a $\kappa<0$

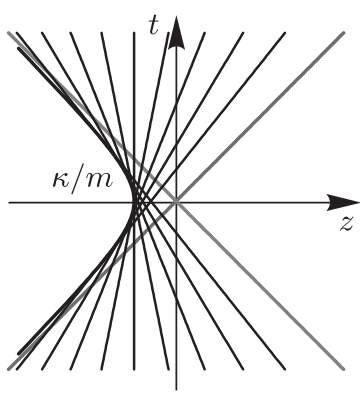

б

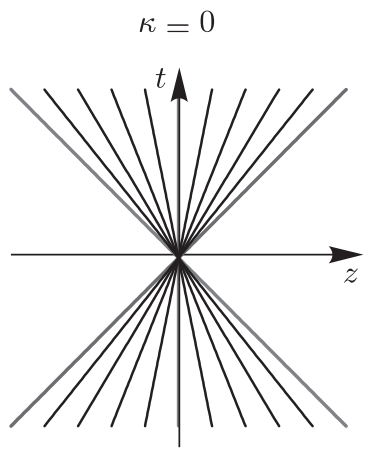

B

Рис. 1. Классические траектории массивных бустовых частиц.

удаляя из спектра точку $\kappa=0$, мы удаляем пучок, состоящий из бесконечного числа траекторий, таким образом теряя существенную часть степеней свободы. Это означает, что точка $\kappa=0$ имеет ненулевую меру в $h_{0}$ и объясняет сингулярность типа дельта-функции у бустовых мод в $h_{0}$.

Точки, лежащие на поверхности светового конуса, требуют отдельного рассмотрения. Прямые $z= \pm t$ не могут быть траекториями массивных частиц. Однако ветви гиперболы (37) при $\kappa \rightarrow 0$ вырождаются в линии, составляющие световой конус в нашей двумерной задаче. Как видно из рис. 1a, 1б, все мировые линии, кроме тех, которые касаются ветвей гиперболы в точке $t=0$, также стремятся к прямым $z= \pm t$ в пределе $\kappa \rightarrow 0$. Таким образом, прямые $z= \pm t$ являются линиями сгущения мировых линий при $\kappa \rightarrow 0$. Возможно, это объясняет наличие сингулярности типа дельта-функции у бустовых мод на световом конусе.

Следует отметить, что существует и другое представление для бустовых мод (20), (21). Имея в виду, что в (20) переменная $t$ имеет бесконечно малую отрицательную мнимую добавку $-i \sigma$, после замены переменной интегрирования $q=\ln u$ получим для $\Psi_{\kappa}^{( \pm)}(x)$ следующее выражение:

$$
\Psi_{\kappa}^{( \pm)}(x)=\frac{1}{2^{3 / 2} \pi} \int_{0}^{\infty} d u u^{-i \kappa-1} e^{-\beta / u-\gamma u},
$$

где $\beta=m\left(\sigma \pm i x_{+}\right) / 2$ и $\gamma=m\left(\sigma \pm i x_{-}\right) / 2$. Далее, с помощью формулы $3.471(9)$ из [50] получаем

$$
\Psi_{\kappa}^{( \pm)}(x)=\frac{1}{\pi \sqrt{2}}\left(\frac{x_{-} \mp i \sigma}{x_{+} \mp i \sigma}\right)^{i \kappa / 2} K_{i \kappa}\left(w_{ \pm}\right), \quad w_{ \pm}=m \sqrt{e^{ \pm i \pi}\left(x_{+} \mp i \sigma\right)\left(x_{-} \mp i \sigma\right)},
$$

где $K_{i \kappa}\left(w_{ \pm}\right)$- функция Макдональда, а обобщенные функции $(\xi \mp i \sigma)^{\lambda}$ следует понимать как (см. [51])

$$
(\xi \mp i \sigma)^{\lambda}=\xi^{\lambda} \theta(\xi)+e^{\mp i \pi \lambda}(-\xi)^{\lambda} \theta(-\xi) .
$$

Используя (41), находим (см. [6], [36])

$$
\begin{aligned}
\Psi_{\kappa}(x)=\theta( & \left.-x_{+}\right) \theta\left(-x_{-}\right) \Psi_{\kappa}^{(\mathrm{P})}(x)+\theta\left(x_{+}\right) \theta\left(-x_{-}\right) \Psi_{\kappa}^{(\mathrm{R})}(x)+ \\
& +\theta\left(x_{+}\right) \theta\left(x_{-}\right) \Psi_{\kappa}^{(\mathrm{F})}(x)+\theta\left(-x_{+}\right) \theta\left(x_{-}\right) \Psi_{\kappa}^{(\mathrm{L})}(x),
\end{aligned}
$$


где

$$
\begin{aligned}
& \Psi_{\kappa}^{(\mathrm{P})}(x)=\frac{i e^{-\pi \kappa / 2}}{2^{3 / 2}}\left(\frac{-x_{-}}{-x_{+}}\right)^{i \kappa / 2} H_{i \kappa}^{(1)}\left(m \sqrt{\left(-x_{+}\right)\left(-x_{-}\right)}\right), \\
& \Psi_{\kappa}^{(\mathrm{R})}(x)=\frac{e^{\pi \kappa / 2}}{\pi \sqrt{2}}\left(\frac{-x_{-}}{x_{+}}\right)^{i \kappa / 2} K_{i \kappa}\left(m \sqrt{x_{+}\left(-x_{-}\right)}\right), \\
& \Psi_{\kappa}^{(\mathrm{F})}(x)=\frac{-i e^{\pi \kappa / 2}}{2^{3 / 2}}\left(\frac{x_{-}}{x_{+}}\right)^{i \kappa / 2} H_{i \kappa}^{(2)}\left(m \sqrt{x_{+} x_{-}}\right), \\
& \Psi_{\kappa}^{(\mathrm{L})}(x)=\frac{e^{-\pi \kappa / 2}}{\pi \sqrt{2}}\left(\frac{x_{-}}{-x_{+}}\right)^{i \kappa / 2} K_{i \kappa}\left(m \sqrt{\left(-x_{+}\right) x_{-}}\right)
\end{aligned}
$$

- выражения для бустовых мод в прошлом (P), правом (R), будущем (F) и левом (L) секторах ПМ соответственно. Здесь $H_{i \kappa}^{(1,2)}(w)$ - функции Ганкеля.

Положив $\kappa=0$ в (43) и учитывая (25), нетрудно воспроизвести результат Вайтмана для $\Delta^{(+)}(x)$ (см. равенство $(4.9)$ в [48]). Поступая таким же образом в $(40)$, получим

$$
\Delta^{( \pm)}(x)= \pm \frac{i}{2 \pi} K_{0}\left(w_{ \pm}\right)
$$

Это новое компактное представление для функции Вайтмана в двумерной скалярной теории.

\section{3. ФЕРМИОНЫ}

Набор ортонормированных положительно- и отрицательно-частотных решений $\left\{F_{a}^{( \pm)}(x)\right\}$ уравнения Дирака в двумерном ПМ

$$
\mathcal{D}_{-} \psi(x)=0, \quad \mathcal{D}_{ \pm}=\left(i \gamma^{0} \frac{\partial}{\partial t}+i \gamma^{1} \frac{\partial}{\partial z} \pm m\right)
$$

где $\gamma^{0,1}$ - двумерные матрицы Дирака, $\gamma^{0} \equiv \beta, \gamma^{0} \gamma^{1}=-\gamma^{1} \gamma^{0} \equiv \alpha, \alpha^{2}=\beta^{2}=1$, полон, если

$$
\int d a F_{a}^{( \pm)}\left(x^{\prime}\right) F_{a}^{( \pm)^{\dagger}}\left(x^{\prime \prime}\right) \gamma^{0}=-i S^{( \pm)}\left(x^{\prime}-x^{\prime \prime}\right)
$$

где положительно-частотные $(+)$ и отрицательно-частотные $(-)$ функции Вайтмана фермионного поля равны

$$
S^{( \pm)}(x)=\mathcal{D}_{+} \Delta^{( \pm)}(x), \quad \Delta^{(-)}(x)=\Delta^{(+)^{*}}(x),
$$

и $\Delta^{(+)}(x)$ определена в $(5)$.

Как и в бозонном случае, любой набор $\left\{F_{a}^{( \pm)}(x)\right\}$ можно построить из набора матриц $\left\{S^{( \pm)}(x-u)\right\}$. Принимая во внимание, что операторы Дирака $\mathcal{D}_{ \pm}$коммутируют с фермионным оператором буста $\mathcal{B}_{\mathcal{D}}$,

$$
\left[\mathcal{D}_{ \pm}, \mathcal{B}_{\mathcal{D}}\right]_{-}=0, \quad \mathcal{B}_{\mathcal{D}}=\mathcal{B}_{\mathcal{K}}-\frac{i}{2} \gamma^{0} \gamma^{1}=i\left(z \partial_{t}+t \partial_{z}-\frac{1}{2} \alpha\right)
$$

нетрудно проверить, что функции

$$
\psi_{\kappa}^{( \pm)}(x)=\int_{-\infty}^{\infty} d q S^{( \pm)}\left(x-u_{q}\right) f_{\kappa}(q)
$$


$\left(u_{q}\right.$ определено в (17)) являются собственными для этого оператора, если

$$
f_{\kappa}(q)=e^{-i \kappa q+\alpha q / 2} C
$$

где $C$ - произвольный постоянный двухкомпонентный столбец. Подставляя (50) в (49), с учетом определения (47) получим следующее выражение для нормированных бустовых мод:

$$
\psi_{\kappa}^{( \pm)}(x)=\frac{1}{2 \pi} \sqrt{\frac{m}{2}} \int_{-\infty}^{\infty} d q e^{\mp i m[(t \mp i \sigma) \operatorname{ch} q-z \operatorname{sh} q]-i \kappa q+\alpha q / 2}(1 \pm \beta) \eta .
$$

Здесь произвольный постоянный столбец $\eta$ удовлетворяет соотношению

$$
\eta^{+}(1 \pm \beta) \eta=1
$$

Прямое сравнение представления (51) с результатами [42]-[44] осложняется тем, что авторы этих работ по неясным причинам используют в двумерном пространстве-времени матрицы размера $4 \times 4$, которые являются приводимыми представлениями матриц Дирака. Переход к такому представлению может быть реализован следующим образом:

$$
\alpha \rightarrow \alpha_{3}=\alpha \otimes \sigma_{3}, \quad \beta \rightarrow \beta \otimes I, \quad \eta \rightarrow \eta \otimes\left(\begin{array}{l}
1 \\
0
\end{array}\right)
$$

где

$$
\alpha=\left(\begin{array}{ll}
0 & 1 \\
1 & 0
\end{array}\right), \quad \beta=\left(\begin{array}{cc}
1 & 0 \\
0 & -1
\end{array}\right), \quad \eta=\frac{1}{\sqrt{2}}\left(\begin{array}{l}
1 \\
1
\end{array}\right) .
$$

В частности, используя обозначения из [42], [43]

$$
v=\frac{1}{2} \ln \frac{x_{+}}{-x_{-}}, \quad u=\sqrt{x_{+}\left(-x_{-}\right)}, \quad \omega \equiv \kappa, \quad \Phi_{\omega}^{ \pm}=H_{i \omega \pm 1 / 2}^{(1)}(i m u),
$$

для положительно-частотного решения в риндлеровском секторе $\mathrm{R}$ двумерного ПМ из (51) с учетом (53), (54) получаем

$$
\psi_{\omega}^{+} \theta\left(x_{+}\right) \theta\left(-x_{-}\right)=\frac{i}{4} \sqrt{m} e^{-i \omega v}\left(\begin{array}{c}
e^{v / 2} \Phi_{\omega}^{-}+e^{-v / 2} \Phi_{\omega}^{+} \\
0 \\
e^{v / 2} \Phi_{\omega}^{-}-e^{-v / 2} \Phi_{\omega}^{+} \\
0
\end{array}\right) \theta\left(x_{+}\right) \theta\left(-x_{-}\right) .
$$

Следует отметить, что результаты [42]-[44] (см. выражения (21.64), (21.65), (21.77) и (21.78a) в [43]) отличаются от (56) отсутствием множителей $e^{ \pm v / 2}$ перед функциями $\Phi_{\omega}^{\mp}$. В рамках метода, используемого авторами [42], [43], эти множители должны были бы появиться при переходе от риндлеровских координат $\{u, v\}$ к декартовым (см., например, [52])

$$
\psi(t, z)=e^{\alpha v / 2} \psi(u, v) .
$$

Кроме того, моды из [42]-[44] отличаются от (56) дополнительным нормировочным множителем $1 / \sqrt{3}$. Эта неточность появилась в результате неверного способа нормировки, использованного авторами [42]-[44]. В самом деле, в этих работах 
нормировочный интеграл в двумерном ПМ представлен в виде суммы четырех слагаемых, каждое из которых равно нормировочному интегралу в одном из четырех секторов ПМ. Однако правильный алгоритм нормировки предполагает интегрирование по некоторой поверхности Коши в ПМ. Наиболее удобная поверхность для этой цели - это прямая $t=0$ (cp., например, [1], [6]). Следовательно, только решения в секторах R и L ПМ вносят вклад в нормировочный интеграл, и нормировочный множитель совпадает с приведенным в (56).

Далее мы будем использовать представление матриц Дирака, отличное от (54),

$$
\gamma^{0} \equiv \beta=\sigma_{1}, \quad \gamma^{1}=i \sigma_{2}, \quad \gamma^{0} \gamma^{1} \equiv \alpha=-\sigma_{3},
$$

где $\sigma_{i}, i=1,2,3,-$ матрицы Паули. Использование представления (58) позволяет существенно упростить вид решений уравнения (45). Как мы увидим ниже, оно также очень удобно для предельного перехода $m \rightarrow 0$. При таком выборе матриц Дирака представление (51) для бустовых мод принимает вид

$$
\psi_{\kappa}^{( \pm)}(x)=\frac{1}{2 \pi} \sqrt{\frac{m}{2}} \int_{-\infty}^{\infty} d q e^{\mp i m((t \mp i \sigma) \operatorname{ch} q-z \operatorname{sh} q)-i \kappa q}\left(\begin{array}{c}
e^{-q / 2} \\
\pm e^{q / 2}
\end{array}\right) .
$$

Используя в точности такую же процедуру, как для скалярных бустовых мод (20), можно привести (59) к виду

$$
\psi_{\kappa}^{( \pm)}(x)=\frac{1}{\pi} \sqrt{\frac{m}{2}}\left(\begin{array}{c}
\left(\frac{x_{-} \mp i \sigma}{x_{+} \mp i \sigma}\right)^{1 / 4+i \kappa / 2} K_{1 / 2+i \kappa}\left(w_{ \pm}\right) \\
\pm\left(\frac{x_{-} \mp i \sigma}{x_{+} \mp i \sigma}\right)^{-1 / 4+i \kappa / 2} K_{1 / 2-i \kappa}\left(w_{ \pm}\right)
\end{array}\right),
$$

где снова $w_{ \pm}=m \sqrt{e^{ \pm i \pi}\left(x_{+} \mp i \sigma\right)\left(x_{-} \mp i \sigma\right)}$.

Из вывода решений (59), (60) уравнения Дирака (45) с очевидностью следует, что полученные решения являются нормированными собственными функциями фермионного оператора буста $\mathcal{B}_{\mathcal{D}}(48)$, со скалярным произведением

$$
(\Phi, \Psi)=\int_{-\infty}^{\infty} d z \Phi^{+}(z, t) \Psi(z, t) .
$$

Используя стандартную аргументацию (см., например, [47], [48]), нетрудно убедиться, что $\mathcal{B}_{\mathcal{D}}$ является самосопряженным оператором, и поэтому его собственные функции образуют полный набор в оснащенном гильбертовом пространстве с положительной нормой $(\Psi, \Psi)>0$. Это утверждение эквивалентно условию полноты (46). Следовательно, корректное квантование массивного фермионного поля в базисе полного набора бустовых мод (59), (60) унитарно-эквивалентно квантованию поля в базисе плоских волн.

В полной аналогии с (42) положительно-частотная бустова мода (60) может быть записана в виде

$$
\begin{aligned}
\psi_{\kappa}^{(+)}(x)=\theta & \left(-x_{+}\right) \theta\left(-x_{-}\right)^{\mathrm{P}} \psi_{\kappa}^{(+)}(x)+\theta\left(x_{+}\right) \theta\left(-x_{-}\right)^{\mathrm{R}} \psi_{\kappa}^{(+)}(x)+ \\
& +\theta\left(x_{+}\right) \theta\left(x_{-}\right)^{\mathrm{F}} \psi_{\kappa}^{(+)}(x)+\theta\left(-x_{+}\right) \theta\left(x_{-}\right)^{\mathrm{L}} \psi_{\kappa}^{(+)}(x),
\end{aligned}
$$


где в секторе $\mathrm{P}$

$$
\mathrm{P}_{\psi_{\kappa}}^{(+)}(x)=\frac{i}{2} \sqrt{\frac{m}{2}} e^{-\pi \kappa / 2+i \pi / 4}\left(\begin{array}{c}
\left(\frac{-x_{-}}{-x_{+}}\right)^{1 / 4+i \kappa / 2} H_{1 / 2+i \kappa}^{(1)}\left(m \sqrt{\left(-x_{+}\right)\left(-x_{-}\right)}\right) \\
-i\left(\frac{-x_{-}}{-x_{+}}\right)^{-1 / 4+i \kappa / 2} H_{-1 / 2+i \kappa}^{(1)}\left(m \sqrt{\left(-x_{+}\right)\left(-x_{-}\right)}\right)
\end{array}\right),
$$

в секторе $\mathrm{R}$

$$
\mathrm{R}_{\psi_{\kappa}}^{(+)}(x)=\frac{1}{\pi} \sqrt{\frac{m}{2}} e^{\pi \kappa / 2-i \pi / 4}\left(\begin{array}{c}
\left(\frac{-x_{-}}{x_{+}}\right)^{1 / 4+i \kappa / 2} K_{1 / 2+i \kappa}\left(m \sqrt{x_{+}\left(-x_{-}\right)}\right) \\
i\left(\frac{-x_{-}}{x_{+}}\right)^{-1 / 4+i \kappa / 2} K_{-1 / 2+i \kappa}\left(m \sqrt{x_{+}\left(-x_{-}\right)}\right)
\end{array}\right),
$$

B cекторе $\mathrm{F}$

$$
\mathrm{F}_{\kappa} \psi_{\kappa}^{(+)}(x)=-\frac{i}{2} \sqrt{\frac{m}{2}} e^{\pi \kappa / 2-i \pi / 4}\left(\begin{array}{c}
\left(\frac{x_{-}}{x_{+}}\right)^{1 / 4+i \kappa / 2} H_{1 / 2+i \kappa}^{(2)}\left(m \sqrt{x_{+} x_{-}}\right) \\
i\left(\frac{x_{-}}{x_{+}}\right)^{-1 / 4+i \kappa / 2} H_{-1 / 2+i \kappa}^{(2)}\left(m \sqrt{x_{+} x_{-}}\right)
\end{array}\right)
$$

и, наконец, в секторе L

$$
{ }^{\mathrm{L}} \psi_{\kappa}^{(+)}(x)=\frac{1}{\pi} \sqrt{\frac{m}{2}} e^{-\pi \kappa / 2+i \pi / 4}\left(\begin{array}{c}
\left(\frac{x_{-}}{-x_{+}}\right)^{1 / 4+i \kappa / 2} K_{1 / 2+i \kappa}\left(m \sqrt{\left(-x_{+}\right) x_{-}}\right) \\
-i\left(\frac{x_{-}}{-x_{+}}\right)^{-1 / 4+i \kappa / 2} K_{-1 / 2+i \kappa}\left(m \sqrt{\left(-x_{+}\right) x_{-}}\right)
\end{array}\right) .
$$

Такое же представление можно привести и для отрицательно-частотных мод $\psi_{\kappa}^{(-)}(x)$.

Бустовы моды (59), (60) ортонормированы условием

$$
\left(\psi_{\kappa^{\prime}}^{( \pm)}, \psi_{\kappa}^{( \pm)}\right)=\int_{-\infty}^{\infty} d z \psi_{\kappa^{\prime}}^{( \pm)^{\dagger}}(x) \psi_{\kappa}^{( \pm)}(x)=\delta\left(\kappa-\kappa^{\prime}\right)
$$

образуют полный набор в смысле (46) и потому могут служить базисом для квантования фермионного поля в ПМ,

$$
\Psi(x)=\int_{-\infty}^{\infty} d \kappa\left(b_{\kappa} \psi_{\kappa}^{(+)}(x)+\tilde{b}_{\kappa}^{+} \psi_{\kappa}^{(-)}(x)\right),
$$

где $b_{\kappa}$ и $\tilde{b}_{\kappa}-$ операторы уничтожения бустовых фермионных частиц и античастиц соответственно, удовлетворяющие стандартным коммутационным соотношениям. Как отмечено выше, по тем же причинам, что и в бозонном случае, бустово квантование фермионного поля унитарно-эквивалентно квантованию в базисе плоских волн

$$
\Psi(x)=\int_{-\infty}^{\infty} d p\left(a_{p} \psi_{p}^{(+)}(x)+\tilde{a}_{p}^{+} \psi_{p}^{(-)}(x)\right)
$$

где

$$
\psi_{p}^{( \pm)}(x)=\left(\begin{array}{c}
\sqrt{\varepsilon_{p}-p} \\
\pm \sqrt{\varepsilon_{p}+p}
\end{array}\right) \frac{e^{\mp i \varepsilon_{p} t \pm i p z}}{\sqrt{4 \pi \varepsilon_{p}}}, \quad \varepsilon_{p}=\sqrt{p^{2}+m^{2}}
$$


Нетрудно убедиться, что бустовы моды (59) связаны с плоскими волнами (69) интегральным преобразованием

$$
\psi_{\kappa}^{( \pm)}(x)=\int_{-\infty}^{\infty} \frac{d p}{\sqrt{2 \pi \varepsilon_{p}}} e^{-i q \kappa} \psi_{p}^{( \pm)}(x), \quad q=\operatorname{arcsh} \frac{p}{m} .
$$

Моды $\psi_{p}^{( \pm)}(x)$ и $\psi_{\kappa}^{( \pm)}(x)$ являются обобщенными функциями по переменным $p$ и $\kappa$. Это означает, что они определяют линейные функционалы

$$
\begin{aligned}
\mathfrak{F}^{( \pm)}(\mathfrak{g}, x) & =\int_{-\infty}^{\infty} d p \psi_{p}^{( \pm)}(x) \mathfrak{g}(p), \\
\mathfrak{F}^{( \pm)}(\mathfrak{h}, x) & =\int_{-\infty}^{\infty} d \kappa \psi_{\kappa}^{( \pm)}(x) \mathfrak{h}(\kappa)
\end{aligned}
$$

на некоторых множествах основных функций $\mathfrak{g}(p)$ и $\mathfrak{h}(\kappa)$ соответственно. Такие функционалы естественно возникают, например, при вычислении матричных элементов операторов поля (67), (68) между вакуумным состоянием $\left|0_{M}\right\rangle$ и состояниями, представляющими волновые пакеты частиц (или античастиц),

$$
\left|1_{\mathfrak{g}}\right\rangle=\int_{-\infty}^{\infty} d p \mathfrak{g}(p) a_{p}^{+}\left|0_{M}\right\rangle, \quad\left|1_{\mathfrak{h}}\right\rangle=\int_{-\infty}^{\infty} d \kappa \mathfrak{h}(\kappa) b_{\kappa}^{+}\left|0_{M}\right\rangle .
$$

Физические одночастичные состояния должны быть нормированы:

$$
\left\langle 1_{\mathfrak{g}} \mid 1_{\mathfrak{g}}\right\rangle=\int_{-\infty}^{\infty} d p|\mathfrak{g}(p)|^{2}=1, \quad\left\langle 1_{\mathfrak{h}} \mid 1_{\mathfrak{h}}\right\rangle=\int_{-\infty}^{\infty} d \kappa|\mathfrak{h}(\kappa)|^{2}=1 .
$$

Следовательно, $\mathfrak{g}(p)$ и $\mathfrak{h}(\kappa)$ должны быть квадратично-интегрируемыми функциями от $p$ и $\kappa$ соответственно. Отсюда нетрудно увидеть, что матричные элементы (71), (72) являются квадратично-интегрируемыми функциями $x$. Ясно, что функционалы (71), (72) - элементы одного и того же функционального пространства. Поэтому существует взаимно однозначное соответствие между основными функциями $\mathfrak{g}(p)$ и $\mathfrak{h}(\kappa)$ :

$$
\mathfrak{h}(\kappa)=\int_{-\infty}^{\infty} \frac{e^{i \kappa q}}{\sqrt{2 \pi \varepsilon_{p}}} \mathfrak{g}(p) d p
$$

где $q=\operatorname{arsh}(p / m)$ - быстрота. Для удобства ограничимся рассмотрением функций $\mathfrak{g}(p)$, принадлежащих классу непрерывных кусочно-гладких функций, убывающих при $|p| \rightarrow \infty$ быстрее, чем $|p|^{-1}$. Это требование гарантирует конечность среднего значения энергии одночастичного состояния $\left|1_{\mathfrak{g}}\right\rangle$ в $(73)$,

$$
E(1)=\int_{-\infty}^{\infty} d p \varepsilon_{p}\left\langle 1_{\mathfrak{g}}\left|a_{p}^{+} a_{p}\right| 1_{\mathfrak{g}}\right\rangle=\int_{-\infty}^{\infty} d p \varepsilon_{p}|\mathfrak{g}(p)|^{2}, \quad \varepsilon_{p}=\sqrt{p^{2}+m^{2}} .
$$

Для установления свойств основных функций $\mathfrak{h}(\kappa)$ воспользуемся соотношением (75).

Сначала покажем на простом примере, что, в отличие от функционала $(71)$, для функционала (72) необходимо знать поведение основных функций $\mathfrak{h}(\kappa)$ не только на вещественной оси, но также и в комплексной плоскости. Рассмотрим функцию

$$
\mathfrak{g}(p)=\frac{e^{-\beta^{2} / 4 \alpha}}{\left(2 \pi \alpha \varepsilon_{p}^{2}\right)^{1 / 4}} e^{-q^{2} / 4 \alpha+\beta q / 2 \alpha}, \quad \alpha>0,
$$


которая, очевидно, удовлетворяет сформулированным выше требованиям на функции $\mathfrak{g}(p)$. Используя $(75)$, получаем

$$
\mathfrak{h}(\kappa)=\left(\frac{2 \alpha}{\pi}\right)^{1 / 4} e^{-\alpha \kappa^{2}+i \beta \kappa} .
$$

Тогда прямое вычисление интеграла (72) в точке $x=0$ дает

$$
\mathfrak{F}^{( \pm)}(\mathfrak{h}, 0)=\sqrt{\frac{m}{2}}\left(\frac{2 \alpha}{\pi}\right)^{1 / 4}\left(\begin{array}{c}
e^{\alpha / 4-\beta / 2} \\
\pm e^{\alpha / 4+\beta / 2}
\end{array}\right)=\sqrt{\frac{m}{2}}\left(\begin{array}{c}
\mathfrak{h}\left(\frac{i}{2}\right) \\
\pm \mathfrak{h}\left(-\frac{i}{2}\right)
\end{array}\right) .
$$

Отсюда видно, что значение функционала (72) в вершине светового конуса определяется значениями основной функции $\mathfrak{h}(\kappa)$ в мнимых точках $\kappa= \pm i / 2$.

Вернемся теперь к соотношению (75). После замены переменной интегрирования $p=m \operatorname{sh} q$ оно может быть записано как преобразование Фурье от функции $\widetilde{\mathfrak{g}}(q)=\sqrt{m \operatorname{ch} q / 2 \pi} \mathfrak{g}(m \operatorname{sh} q)$ :

$$
\mathfrak{h}(\kappa)=\int_{-\infty}^{\infty} d q e^{i \kappa q} \widetilde{\mathfrak{g}}(q)
$$

В рамках наших допущений

$$
\widetilde{\mathfrak{g}}(q)=O\left(e^{-(1 / 2+\epsilon)|q|}\right), \quad \epsilon>0,
$$

при $|q| \rightarrow \infty$. Тогда в соответствии с теоремой Пэли-Винера [53] основные функции $\mathfrak{h}(\kappa)(80)$ являются аналитическими в полосе

$$
-\frac{1}{2}-\epsilon<\operatorname{Im} \kappa<\frac{1}{2}+\epsilon, \quad \epsilon>0 .
$$

Вычислим интеграл в (80) по частям. Так как основные функции $\widetilde{\mathfrak{g}}(q)$ непрерывны и экспоненциально убывают при $|q| \rightarrow \infty$ (см. (81)), внеинтегральный член обращается в нуль. Следовательно, функции $\mathfrak{h}(\kappa)$ в своей области аналитичности $(82)$ убывают при $|\kappa| \rightarrow \infty$ по крайней мере как $\kappa^{-2}$. Это означает, что путь интегрирования в (72) может быть сдвинут в пределах полосы (82).

Рассмотрим функционал (72) в точке $x=0$. Сдвинув путь интегрирования для верхней компоненты на $i / 2$ вверх, а для нижней - на $i / 2$ вниз, получим

$$
\begin{aligned}
\mathfrak{F}^{( \pm)}(\mathfrak{h}, 0) & =\frac{1}{2 \pi} \sqrt{\frac{m}{2}} \int_{-\infty}^{\infty} d \kappa d q e^{-i \kappa q}\left(\begin{array}{c}
\mathfrak{h}\left(\kappa+\frac{i}{2}\right) \\
\pm \mathfrak{h}\left(\kappa-\frac{i}{2}\right)
\end{array}\right)= \\
& =\sqrt{\frac{m}{2}} \int_{-\infty}^{\infty} d \kappa \delta(\kappa)\left(\begin{array}{c}
\mathfrak{h}\left(\kappa+\frac{i}{2}\right) \\
\pm \mathfrak{h}\left(\kappa-\frac{i}{2}\right)
\end{array}\right)=\sqrt{\frac{m}{2}}\left(\begin{array}{c}
\mathfrak{h}\left(\frac{i}{2}\right) \\
\pm \mathfrak{h}\left(-\frac{i}{2}\right)
\end{array}\right) .
\end{aligned}
$$




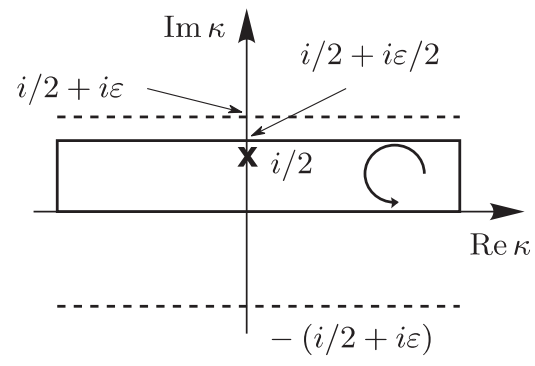

a

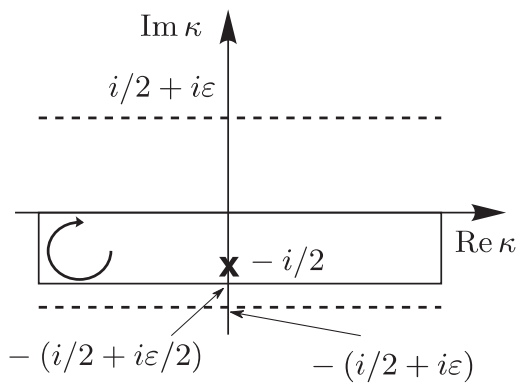

6

Рис. 2. Контуры интегрирования для верхней (а) и нижней (б) компонент $\mathfrak{F}^{( \pm)}(\mathfrak{h}, 0)$.

Соотношение (83) обобщает формулу (79) на класс функций $\mathfrak{h}(\kappa)$, аналитических в полосе (82) и достаточно быстро убывающих при $|\kappa| \rightarrow \infty$.

Полученный результат позволяет записать фермионные бустовы моды в вершине светового конуса через дельта-функции комплексного аргумента:

$$
\psi_{\kappa}^{( \pm)}(0)=\sqrt{\frac{m}{2}}\left(\begin{array}{c}
\delta\left(\kappa-\frac{i}{2}\right) \\
\pm \delta\left(\kappa+\frac{i}{2}\right)
\end{array}\right) .
$$

Функционалы $\mathfrak{F}^{( \pm)}(\mathfrak{h}, 0)$ можно также представить в виде интегралов по замкнутым контурам, изображеным на рис. 2а, 2б:

$$
\mathfrak{F}^{( \pm)}(\mathfrak{h}, 0)=\oint_{C_{a, b}} d \kappa \psi_{\kappa}^{( \pm)}(0) \mathfrak{h}(\kappa)
$$

Действительно, явный вид $\psi_{\kappa}^{( \pm)}(0)$ можно легко вывести из равенства (60). Полагая $x_{+}=x_{-}=0$ и используя разложение в ряд для $K_{\nu}(z)$ [50], имеем

$$
\psi_{\kappa}^{( \pm)}(0)=\lim _{\sigma \rightarrow 0} \frac{1}{2 \pi}\left(\begin{array}{c}
\left(\frac{m}{2}\right)^{-i \kappa} \frac{\Gamma(1 / 2+i \kappa)}{\sigma^{1 / 2+i \kappa}} \\
\pm\left(\frac{m}{2}\right)^{i \kappa} \frac{\Gamma(1 / 2-i \kappa)}{\sigma^{1 / 2-i \kappa}}
\end{array}\right) .
$$

Очевидно, что интегралы по вертикальным отрезкам контуров $C_{a, b}$ равны нулю. Интегралы по нижней $\left(C_{a}\right)$ и верхней $\left(C_{b}\right)$ частям контура также равны нулю в пределе $\sigma \rightarrow 0$. Это утверждение нетрудно проверить прямым вычислением. Далее, учитывая, что подынтегральное выражение в (85) имеет полюсы первого порядка в точках $\kappa= \pm i / 2$, и пользуясь теоремой Коши о вычетах, сразу воспроизводим результат (83).

Дельта-функция комплексного аргумента была впервые введена Гельфандом и Шиловым в [51] на основании теоремы о вычетах Коши,

$$
\left(\delta\left(\kappa-\kappa_{0}\right), f(\kappa)\right)=\frac{1}{2 \pi i} \oint_{L} \frac{f(\kappa) d \kappa}{\kappa-\kappa_{0}}=f\left(\kappa_{0}\right),
$$


где $L$ - замкнутый контур вокруг произвольной комплексной точки $\kappa_{0}$. Обобщенная функция (87) определена в [51] на классе целых функций $f(\kappa)$, так называемом классе $Z$. Наш класс основных функций, аналитических в полосе (82), отличается от $Z$. Поэтому некоторые свойства дельта-функции Гельфанда [51] в нашем случае могут не выполняться. Тем не менее обратное утверждение справедливо: все свойства нашей дельта-функции справедливы и для дельта-функции Гельфанда.

Итак, как на классе основных функций, аналитических в полосе (82), так и для функций на классе $Z$ верно следующее равенство обобщенных функций [41]:

$$
\lim _{\sigma \rightarrow 0} \frac{1}{2 \pi} \frac{\Gamma(1 / 2 \pm i \kappa)}{\sigma^{1 / 2 \pm i \kappa}}=\delta\left(\kappa \mp \frac{i}{2}\right) .
$$

Покажем теперь, что моды (59), (60) обладают сингулярностями типа дельтафункции не только в вершине светового конуса, но также и на линиях $x_{ \pm}=0$. Действительно, на поверхности светового конуса аргументы функций Макдональда в (60) малы, $\left|w_{ \pm}\right| \ll 1$. Поэтому, используя разложение в ряд для функции Макдональда [50], получаем

$$
\begin{aligned}
\psi_{\kappa}^{( \pm)}(x)=\frac{e^{ \pm \pi \kappa / 2 \mp i \pi / 4}}{2 \pi} \times \\
\times\left(\begin{array}{c}
\left(\frac{m}{2}\right)^{-i \kappa} \frac{\Gamma(1 / 2+i \kappa)}{\left(x_{+} \mp i \sigma\right)^{1 / 2+i \kappa}}+\left(\frac{m}{2}\right)^{i \kappa+1} \frac{\Gamma(-1 / 2-i \kappa)}{\left(-x_{-} \pm i \sigma\right)^{-1 / 2-i \kappa}} \\
i\left(\frac{m}{2}\right)^{i \kappa} \frac{\Gamma(1 / 2-i \kappa)}{\left(-x_{-} \pm i \sigma\right)^{1 / 2-i \kappa}}+i\left(\frac{m}{2}\right)^{-i \kappa+1} \frac{\Gamma(-1 / 2+i \kappa)}{\left(x_{+} \mp i \sigma\right)^{-1 / 2+i \kappa}}
\end{array}\right) .
\end{aligned}
$$

Таким образом, видно, что на образующих светового конуса функции $\psi_{\kappa}^{( \pm)}(x)$ содержат $\delta(\kappa-i / 2)$ при $x_{+}=0$ в верхней и $\delta(\kappa+i / 2)$ при $x_{-}=0$ в нижней компонентах.

В разделе 2 было показано, что в случае скалярного поля семейство бустовых мод после исключения нулевой моды не образует полный набор в ПМ. Это связано с тем, что скалярные бустовы моды обладают особенностью вида дельта-функции на поверхности светового конуса, и поэтому точка $\kappa=0$ дает конечный вклад в значения физических величин. Некоторые из них, такие как, например, функция Вайтмана, целиком определяются спектральной точкой $\kappa=0$. Возникает вопрос, можно ли удалить из спектра точку $\kappa=0$ в фермионном случае. Для выяснения этого вопроса рассмотрим интеграл

$$
\begin{aligned}
\widetilde{\mathfrak{F}}^{( \pm)}(\mathfrak{h}, 0) & =\lim _{\delta \rightarrow 0}\left(\int_{-\infty}^{\kappa_{0}-\delta}+\int_{\kappa_{0}+\delta}^{\infty}\right) d \kappa \psi_{\kappa}^{( \pm)}(0) \mathfrak{h}(\kappa)= \\
& =\mathfrak{F}^{( \pm)}(\mathfrak{h}, 0)-\lim _{\delta \rightarrow 0} \int_{\kappa_{0}-\delta}^{\kappa_{0}+\delta} d \kappa \psi_{\kappa}^{( \pm)}(0) \mathfrak{h}(\kappa),
\end{aligned}
$$

где $\kappa_{0}-$ произвольное действительное число.

Рассмотрим верхнюю компоненту второго слагаемого в правой части (90)

$$
\frac{1}{2 \pi} \lim _{\delta \rightarrow 0} \lim _{\sigma \rightarrow 0} \int_{\kappa_{0}-\delta}^{\kappa_{0}+\delta} d \kappa\left(\frac{m}{2}\right)^{-i \kappa} \frac{\Gamma(1 / 2+i \kappa)}{\sigma^{1 / 2+i \kappa}} \mathfrak{h}(\kappa) .
$$


Интеграл в (91) можно легко вычислить в пределе $\sigma \rightarrow 0$. Имеем

$$
\begin{aligned}
\lim _{\delta \rightarrow 0} \int_{\kappa_{0}-\delta}^{\kappa_{0}+\delta} d \kappa\left[\psi_{\kappa}^{( \pm)}(0)\right]_{\mathrm{up}} \mathfrak{h}(\kappa)= \\
=\frac{i}{2 \pi}\left(\frac{m}{2}\right)^{-i \kappa_{0}} \lim _{\delta \rightarrow 0} \lim _{\sigma \rightarrow 0} \frac{\sigma^{-i \kappa_{0}-1 / 2}}{\ln \sigma}\left[\left(\frac{m}{2}\right)^{-i \delta} \Gamma\left(\frac{1}{2}+i \kappa_{0}+i \delta\right) \mathfrak{h}\left(\kappa_{0}+\delta\right) \sigma^{-i \delta}-\right. \\
\left.\quad-\left(\frac{m}{2}\right)^{i \delta} \Gamma\left(\frac{1}{2}+i \kappa_{0}-i \delta\right) \mathfrak{h}\left(\kappa_{0}-\delta\right) \sigma^{i \delta}+O\left(\frac{1}{\ln \sigma}\right)\right]
\end{aligned}
$$

Выражение (92) ясно демонстрирует, что результат вычисления для $\widetilde{\mathfrak{F}}_{\text {up }}^{( \pm)}(\mathfrak{h}, 0)$ сильно зависит от порядка перехода к пределам $\delta \rightarrow 0$ и $\sigma \rightarrow 0$. Если поменять порядок пределов в $(91),(92)$, мы вернемся от $\widetilde{\mathfrak{F}}^{( \pm)}(\mathfrak{h}, 0)(90)$ обратно к $\mathfrak{F}^{( \pm)}(\mathfrak{h}, 0)(72)$. Неудивительно, что в этом случае (90) воспроизводит результат (83). Принятый нами в (92) порядок пределов соответствует вычислению функционала (90) и приводит к не имеющему смысла результату. Это означает, что в фермионном случае незаконно выкалывание любой точки вещественной оси $\kappa$. Другими словами, набор бустовых мод (59), (60) перестает быть полным в ПМ после удаления из спектра единственной произволъной точки. Он может быть полным только в ПМ без светового конуса (т. е. в четырех не связанных секторах ПМ). В этом смысле наше заключение повторяет аналогичный результат для бозонного поля, который обсуждался в разделе 2 .

Отметим, что последний результат был получен при минимально возможных ограничениях на основные функции $\mathfrak{g}(p)$. Если мы ограничимся функциями $\mathfrak{g}(p)$, принадлежащими классу $K$ гладких функций с компактным носителем, как это обычно делается в КТП, то функции $\mathfrak{h}(\kappa)$ будут принадлежать классу $Z$ [51]. В этом случае доказательство невозможности удаления из спектра произвольной точки $\kappa_{0}$ особенно простое. Действительно, дельта-функция Гельфанда является аналитической обобщенной функцией во всей комплексной плоскости $\kappa$. Следовательно, она может быть разложена в ряд Тейлора в окрестности любой точки $\kappa-\kappa_{0}$,

$$
\delta\left(\kappa \mp \frac{i}{2}\right)=\sum_{n=0}^{\infty} \frac{1}{n !}\left(\kappa_{0} \mp \frac{i}{2}\right)^{n} \delta^{(n)}\left(\kappa-\kappa_{0}\right),
$$

и радиус сходимости ряда (93) равен бесконечности [51]. Здесь $\delta^{(n)}\left(\kappa-\kappa_{0}\right)$ обозначает $n$-ю производную дельта-функции Дирака в точке $\kappa-\kappa_{0}$. В результате получим

$$
\begin{aligned}
& \lim _{\delta \rightarrow 0}\left(\int_{-\infty}^{\kappa_{0}-\delta}+\int_{\kappa_{0}+\delta}^{\infty}\right) d \kappa \delta\left(\kappa \mp \frac{i}{2}\right) \mathfrak{h}(\kappa)= \\
& \quad=\sum_{n=0}^{\infty} \frac{1}{n !}\left(\kappa_{0} \mp \frac{i}{2}\right)^{n} \lim _{\delta \rightarrow 0}\left(\int_{-\infty}^{\kappa_{0}-\delta}+\int_{\kappa_{0}+\delta}^{\infty}\right) d \kappa \delta^{(n)}\left(\kappa-\kappa_{0}\right) \mathfrak{h}(\kappa)=0,
\end{aligned}
$$

и, следовательно, на классе $Z$ основных функций удаление из спектра произвольной точки $\kappa_{0}$ приводит к обращению в нуль функционалов $\mathfrak{F}^{( \pm)}(\mathfrak{h}, 0)(85)$ в вершине светового конуса. В остальных точках поверхности конуса функционалы $\mathfrak{F}^{( \pm)}(\mathfrak{h}, 0)$ изменяются на конечную величину. Однако, поскольку дельта-функция Гельфанда 
аналитическая во всей комплексной плоскости $\kappa$, ее можно разложить в ряд Тейлора в окрестности другой действительной точки $\kappa=\kappa_{1}, \kappa_{1} \neq \kappa_{0}$. Если вычислять интеграл (94), используя представление (93) для дельта-функции, то ясно, что удаление из спектра точки $\kappa_{0}$ не повлияет на результат интегрирования. Поскольку значение матричного элемента (85) зависит от способа вычисления, заключаем, что операция исключения произвольной точки из спектра бессмысленна, что согласуется с предыдущим рассмотрением (см. обсуждение формулы (90) в предыдущем абзаце).

Как было показано в разделе 2, функции Вайтмана $\Delta^{( \pm)}(x)$ массивного скалярного поля определяются нулевыми бустовыми модами $\Psi_{0}^{( \pm)}(x)$ (см. (25)). Это следствие трансляционной инвариантности $\Delta^{( \pm)}(x)$ и наличия в $\Psi_{\kappa}^{( \pm)}(x)$ на световом конусе особенности типа дельта-функции Дирака. Такой же результат верен и для функций Вайтмана массивного фермионного поля. Действительно, вследствие трансляционной инвариантности можно записать (ср. с (46))

$$
S^{( \pm)}\left(x^{\prime}, x^{\prime \prime}\right)=i \int_{-\infty}^{\infty} d \kappa \psi_{\kappa}^{( \pm)}(x) \psi_{\kappa}^{( \pm)^{\dagger}}(0) \gamma^{0}, \quad x=x^{\prime}-x^{\prime \prime}
$$

Используя теперь (84), приходим к следующему результату для матричных элементов $S_{\alpha \beta}^{( \pm)}(x)$ функции Вайтмана $(95)$ :

$$
S_{\alpha 1}^{( \pm)}(x)= \pm i \sqrt{\frac{m}{2}} \psi_{i / 2, \alpha}^{( \pm)}(x), \quad S_{\alpha 2}^{( \pm)}(x)=i \sqrt{\frac{m}{2}} \psi_{-i / 2, \alpha}^{( \pm)}(x), \quad \alpha=1,2,
$$

где $\psi_{\kappa, \alpha}^{( \pm)}(x)-\alpha$-компоненты бустовых мод (59), (60). Таким образом, видно, что матричные элементы $S^{( \pm)}(x)$ определяются только двумя "спектральными точками" $\kappa= \pm i / 2$. В точности так же, как и в скалярном случае, нетрудно убедиться, что этот результат справедлив и для сглаженных функций Вайтмана.

С помощью (60) можно выразить функции Вайтмана (96) в виде (ср. с (44))

$$
S^{( \pm)}(x)=i \frac{m}{2 \pi}\left(\begin{array}{cc} 
\pm K_{0}\left(w_{ \pm}\right) & \left(\frac{x_{+} \mp i \sigma}{x_{-} \mp i \sigma}\right)^{-1 / 2} K_{1}\left(w_{ \pm}\right) \\
\left(\frac{x_{+} \mp i \sigma}{x_{-} \mp i \sigma}\right)^{1 / 2} K_{1}\left(w_{ \pm}\right) & \pm K_{0}\left(w_{ \pm}\right)
\end{array}\right)
$$

где $w_{ \pm}$те же, что и в $(40)$.

Рассмотрим теперь случай безмассового фермионного поля, который впервые исследовался в работе [41]. Функции Вайтмана безмассового поля $S_{0}^{( \pm)}(x)$ легко могут быть получены путем перехода к пределу $m \rightarrow 0$ в формуле (97):

$$
S_{0}^{( \pm)}(x)=\lim _{m \rightarrow 0} S^{( \pm)}(x)= \pm \frac{1}{2 \pi}\left(\begin{array}{cc}
0 & \frac{1}{x_{+} \mp i \sigma} \\
\frac{1}{x_{-} \mp i \sigma} & 0
\end{array}\right), \quad \sigma \rightarrow 0 .
$$

Как и в рассмотренных ранее случаях, можно использовать $S_{0}^{( \pm)}\left(x-\alpha_{q}\right)$ для получения любого полного набора ортонормированных решений уравнения Дирака. 
В частности, для бустовых мод имеем

$$
\chi_{\kappa}^{( \pm)}(x)=\int_{-\infty}^{\infty} d q S_{0}^{( \pm)}\left(x-\alpha_{q}\right) e^{-i \kappa q-q \sigma_{3} / 2} C,
$$

где $C=\left(\begin{array}{l}c_{1} \\ c_{2}\end{array}\right)$ - произвольный двухкомпонентный столбец (ср. (49), (50)). Видно, что при $m=0$ функция Вайтмана (98) антидиагональна. Благодаря этому свойству верхняя и нижняя компоненты бустовой моды (99) становятся независимыми:

$$
\chi_{\kappa}^{( \pm)}(x)=\tilde{c}_{1} \chi_{\kappa+}^{( \pm)}(x) e_{+}+\tilde{c}_{2} \chi_{\kappa-}^{( \pm)}(x) e_{-},
$$

где $e_{ \pm}$являются собственными векторами матрицы Паули $\sigma_{3}, \sigma_{3} e_{ \pm}= \pm e_{ \pm}$. Это связано с тем, что в безмассовом случае появляется новый закон сохранения - закон сохранения киральности (см., например, [54]). В связи с этим решения уравнения Дирака (45) нумеруются дополнительным киральным квантовым числом $\tau= \pm$. Коэффициенты $\tilde{c}_{1}$ и $\tilde{c}_{2}$ в (100) независимы и могут по отдельности быть определены из условия нормировки для решений $\chi_{\kappa+}^{( \pm)}(x) e_{+}$и $\chi_{\kappa-}^{( \pm)}(x) e_{-}$. Отметим, что, поскольку в массивном случае киральность не сохраняется, компоненты столбца $C$ в (50) по отдельности определены быть не могут. В массивном случае их комбинация собирается в общий множитель решения $\psi_{\kappa}^{( \pm)}(x)$ в $(51)$.

Для нормированных положительно-частотных функций $\chi_{\kappa \tau}^{( \pm)}$имеем

$$
\chi_{\kappa \tau}^{( \pm)}(x)=\frac{e^{\mp i \pi \lambda / 2} \Gamma(\lambda)}{2 \pi\left(x_{\tau} \mp i \sigma\right)^{\lambda}}, \quad \lambda=\frac{1}{2}+i \tau \kappa, \quad \sigma \rightarrow 0,
$$

где фазовые множители выбраны из соображений удобства. Следует отметить, что отрицательно-частотные функции $\chi_{\kappa \tau}^{(-)}$получаются из положительно-частотных функций $\chi_{\kappa \tau}^{(+)}$комплексным сопряжением и заменой $\kappa \rightarrow-\kappa$.

Плоские волны в безмассовом случае также нумеруются квантовым числом $\tau$ :

$$
\varphi_{p \tau}^{( \pm)}(x)=\frac{1}{\sqrt{2 \pi}} e^{\mp i p x_{\tau}}, \quad 0<p<\infty .
$$

Они связаны с бустовыми модами $\chi_{\kappa \tau}^{( \pm)}(x) e_{\tau}$ преобразованием Меллина

$$
\chi_{\kappa \tau}^{( \pm)}\left(x_{\tau}\right)=\int_{0}^{\infty} \frac{d p}{\sqrt{2 \pi}} p^{\lambda-1} \varphi_{p \tau}^{( \pm)}\left(x_{\tau}\right)
$$

Это означает, что если мы считаем, что обобщенные функции $\varphi_{p \tau}^{( \pm)}\left(x_{\tau}\right)$ определены на том же классе основных функций $\mathfrak{g}(p)$, что и в массивном случае, то обобщенные функции $\chi_{\kappa \tau}^{( \pm)}\left(x_{\tau}\right)$ будут определены на классе основных функций $\mathfrak{h}(\kappa)$, аналитических в полосе $(82)$ и убывающих при $|\kappa| \rightarrow \infty$ в этой полосе.

Тогда с учетом (88) заключаем, что бустовы моды безмассового фермионного поля на поверхности светового конуса являются дельта-функциями комплексного аргумента (ср. с [41]),

$$
\chi_{\kappa \tau}^{( \pm)}\left(x_{\tau}=0\right)=\delta\left(\kappa-\tau \frac{i}{2}\right)
$$


Покажем теперь, как моды (101) могут быть получены из (60) переходом к пределу $m \rightarrow 0$. Используя разложение в ряд [50] для функций $K_{1 / 2 \pm i \kappa}\left(w_{ \pm}\right)$, получаем

$$
\psi_{\kappa}^{( \pm)}(x)=\left(\frac{m}{2}\right)^{-i \kappa} \chi_{\kappa+}^{( \pm)} e_{+} \pm\left(\frac{m}{2}\right)^{i \kappa} \chi_{\kappa-}^{( \pm)} e_{-},
$$

т. е. представление (100) с коэффициентами $\tilde{c}_{1}$ и $\tilde{c}_{2}$, содержащими сингулярные при $m \rightarrow 0$ фазовые множители. Тем не менее эти множители не влияют ни на нормировочные постоянные, ни на другие физические величины, а потому могут быть опущены.

\section{4. ЗАКЛЮЧИТЕЛЬНЫЕ ЗАМЕЧАНИЯ}

Мы показали, что функция Вайтмана свободного квантованного поля порождает любой полный набор решений релятивистских волновых уравнений. Используя этот подход, мы построили полные наборы решений уравнений КФГ и Дирака, состоящие из собственных функций генератора преобразований Лоренца (оператора буста).

Бустовы моды очень редко используются в качестве базиса для квантования поля. До сих пор их применяли только для анализа так называемого эффекта Унру [1], [6] и при квантовании заряженного массивного скалярного поля в присутствии внешнего постоянного электрического поля [55]-[57]. Тем не менее существует много задач, особенно в КТП в искривленном пространстве, где бустова симметрия является единственной симметрией квантованного поля, и поэтому ее использование для разделения переменных в классическом полевом уравнении остается единственным инструментом для решения таких уравнений.

Специфической особенностью бустовых мод является то, что на поверхности светового конуса они, как функции бустового квантового числа $\kappa$, обладают сильными сингулярностями. Физической причиной этих сингулярностей, несомненно, является сингулярность преобразований Лоренца при $v=c$.

Для случая скалярного поля это сингулярность вида дельта-функции Дирака $\left.\Psi_{\kappa}(x)\right|_{x_{ \pm}=0} \sim \delta(\kappa)[6]$. Это приводит к особой роли нулевой бустовой моды: ее удаление из набора бустовых мод делает его неполным. Исключительная роль нулевой бустовой моды становится ясной, если вспомнить, что при нулевом $\kappa$ функция $\Psi_{0}(x)$ является лоренц-инвариантным положительно-частотным решением уравнения КФГ, т. е. функцией Вайтмана квантованного поля, совпадающей с положительно-частотной частью коммутатора двух операторов скалярного поля. Следовательно, исключение нулевой бустовой моды приводит к "квантовой" теории с коммутирующими полевыми операторами.

Сингулярности фермионных бустовых мод еще сильнее. В данной статье показано, что на поверхности светового конуса их компоненты содержат дельта-функции комплексного аргумента $\left.\psi_{\kappa \alpha}(x)\right|_{x_{ \pm}=0} \sim \delta(\kappa \mp i / 2)$. Дельта-функция комплексного аргумента была впервые введена Гельфандом и Шиловым в [51] и была определена на классе целых функций $Z$. Полученная нами дельта-функция определена на классе основных функций, аналитических в полосе (82). При этом ширина полосы определяется физическими требованиями. Наш выбор обеспечивает квадратичную интегрируемость одночастичных волновых пакетов и конечность их энергии. Если дополнительно потребовать конечность квадрата энергии, то нужно сузить класс основных функций, увеличив ширину полосы до

$$
-\frac{3}{2}-\epsilon<\operatorname{Im} \kappa<\frac{3}{2}+\epsilon, \quad \epsilon>0 .
$$


Дальнейшее ужесточение требований к физическим состояниям приводит к соответствующему увеличению ширины полосы аналитичности основных функций. Поэтому имеет смысл обобщить понятие дельта-функции комплексного аргумента и ввести обобщенную функцию $\delta_{s}(\kappa)$,

$$
\left(\delta_{s}\left(\kappa-\kappa_{0}\right), f(\kappa)\right)=f\left(\kappa_{0}\right)
$$

определенную на классе $Z_{s}$ основных функций $f(\kappa)$, аналитических в полосе

$$
-s-\epsilon<\operatorname{Im} \kappa<s+\epsilon, \quad s>0, \quad \epsilon>0,
$$

где $\kappa_{0}$ лежит внутри полосы. В этих обозначениях дельта-функция, введенная в разделе 3 , будет выглядеть как $\delta_{1 / 2}(\kappa)$, дельта-функция Гельфанда - как $\delta_{\infty}(\kappa)$, а обычная дельта-функция Дирака - как $\delta_{0}(\kappa)$.

Следует отметить, что для дельта-функции, определенной на классе $Z_{s}$, справедливо интегральное представление

$$
\delta_{s}(\nu)=\frac{1}{2 \pi} \int_{-\infty}^{\infty} d q e^{-i \nu q}, \quad \nu=\kappa \mp i s, \quad s>0,
$$

которое является непосредственным обобщением стандартного представления дельта-функции Дирака. Кроме того, справедливы соотношения

$$
\delta_{s}(\nu)=\frac{i}{2} H_{i \nu}^{(1)}(0)=-\frac{i}{2} H_{i \nu}^{(2)}(0)=\frac{1}{\pi} K_{i \nu}(0), \quad \nu=\kappa \mp i s, \quad s>0
$$

(cp. с [58] для случая $s=0)$.

Наличие дельта-функции комплексного аргумента в бустовых модах на поверхности светового конуса не позволяет исключать никакую точку из бустового спектра. Мы показали это для случая $s=1 / 2$ (см. (90)-(94)), но доказательство этого утверждения не меняется для произвольного значения $s$. Другой способ доказательства этого утверждения был использован для обобщенной функции $\delta_{\infty}(\kappa)$ и основан на ее аналитичности во всей комплексной плоскости. Аналогичное доказательство также может быть приведено для дельта-функции $\delta_{s}$. В этом случае из-за конечного значения радиуса сходимости $R=s+\epsilon$ разложение (93) может быть применено только для вещественных $\kappa_{0}=b_{1},\left|b_{1}\right|<\epsilon$. На следующем шаге дельта-функция Дирака $\delta_{0}\left(\kappa-b_{1}\right)$ и любая ее производная могут быть представлены рядом Тейлора вида (93) вокруг точки $b_{2}$ на вещественной оси, $\left|b_{2}-b_{1}\right|<s+\epsilon$. Таким образом, функция $\delta_{s}(\kappa-i s)$ будет разложена в двукратный ряд по дельта-функции Дирака $\delta_{0}\left(\kappa-b_{2}\right)$ и ее производным. После конечного числа шагов $N$ можно достичь произвольной спектральной точки $\kappa_{0}$ и таким образом получить представление $\delta_{s}(\kappa-i s)$ в виде $N$-кратного ряда по дельта-функции Дирака $\delta_{0}\left(\kappa-\kappa_{0}\right)$ и ее производным. Ясно, что тогда точка $\kappa_{0}$ не может быть исключена из спектра.

Это рассуждение ясно показывает, почему скалярный случай, когда имеется только одна выделенная точка $\kappa=0$, которая не может быть исключена из спектра, существенно отличается от фермионного. Действительно, полоса аналитичности (107) для $\delta_{s}(\kappa)$ при $s=0$ вырождается в вещественную ось комплексной плоскости $\kappa$, и поэтому радиус сходимости соответствующего ряда Тейлора становится равным нулю, или, другими словами, дельта-функция Дирака $\delta_{0}(\kappa)$ не является аналитической обобщенной функцией. Поэтому описанная выше процедура не может быть реализована. 
Итак, процедура Унру не может подтвердить существование эффекта Унру ни для бозонного, ни для фермионного поля. Причиной этого является неполнота набора мод Унру в ПМ. В то же время набор мод Унру полон и может служить для квантования поля только в двойном риндлеровском клине $\mathrm{R} \cup \mathrm{L}$, который может быть вырезан из ПМ наложением нетривиального граничного условия на горизонтах [6], [7]. Поле в такой задаче не имеет ничего общего с полем в ПМ, несмотря на то что локально эти поля являются решениями одних и тех же лоренц-инвариантных уравнений КФГ и Дирака.

Отметим, что наши аргументы были основаны на стандартной формулировке КТП. Кроме нее существует другой, так называемый алгебраический подход к КТП (см., например, [59]). С точки зрения алгебраического подхода рассматриваемые "тепловые свойства Унру" эквивалентны свойствам периодичности функций Грина и Вайтмана в мнимом времени. Наиболее точная и строгая формулировка этой идеи дается теоремой Бизоньяно-Вичмана (БВ), которая утверждает, что алгебраическое вакуумное состояние ПМ, ограниченное на алгебру полей, локализованных в двойном риндлеровском клине, удовлетворяет условию Кубо-Мартина-Швингера по отношению к риндлеровскому времени. Ранее в работах [6], [40] мы показали, что теорема БВ неприменима для рассмотрения задачи Унру в случае скалярного поля. Случай фермионного поля в этом смысле практически не отличается от бозонного. Поэтому здесь мы не обсуждаем этот аспект задачи.

Начиная с работы Унру [1] существует мнение, что эффект Унру является точным аналогом процесса Хокинга для вечных черных дыр. Однако эти задачи очень разные. Излучение Хокинга является динамическим процессом рождения пар гравитационным полем. Эффект Унру, если бы он существовал, был бы универсальным тепловым откликом равномерно ускоренного детектора на действие ускоряющей силы. Это означает, что ускоряющая сила не вызывает возбуждений в квантовом поле, которое остается в стабильном вакуумном состоянии, а возбуждает детектор. Поэтому, в отличие от эффекта Хокинга, отклик детектора в задаче Унру может рассматриваться в "аспекте частиц" только как кинематический эффект. Впрочем, неуниверсальность эффекта Унру, продемонстрированная в работах [23], [24], [26], делает рассмотрение такой задачи лишенным смысла и в “аспекте частиц".

Формально общность двух задач связана с тем, что структуры максимально расширенного пространства-времени Шварцшильда (МРПШ) и ПМ выглядят очень похоже (см., например, [60]). В частности, оба пространства могут быть разделены на четыре сектора, ограниченных горизонтами событий. Однако метрика Шварцшильда содержит физическую сингулярность в аналогах секторов Р и F ПМ. Это обстоятельство определяет существенную разницу между двумя пространствами. Поскольку неизвестно, как решения квантовых уравнений ведут себя в окрестности сингулярности, то мы не можем построить КТП во всем МРПШ и проанализировать задачу Хокинга так же, как сделали это в ПМ. Поэтому из факта отсутствия эффекта Унру нельзя вывести аналогичное утверждение относительно эффекта Хокинга. K счастью, есть и другой подход к задаче Хокинга, основанный на втором начале термодинамики, которое занимает, по выражению Эддингтона [61], "высшее положение среди законов природы". 
ПРИЛОЖЕНИЕ А

\section{О вкладе точки $\kappa=0$ в функцию Вайтмана для сглаженных мод}

Рассмотрим более детально сглаженную версию (30) функции Вайтмана скалярного поля

$$
\Delta^{(+)}(f, g)=\int d^{2} x^{\prime} d^{2} x^{\prime \prime} f^{*}\left(x^{\prime}\right) g\left(x^{\prime \prime}\right) \Delta^{(+)}\left(x^{\prime}-x^{\prime \prime}\right)=i \int_{-\infty}^{\infty} d \kappa \Psi_{\kappa}^{(+)}(f) \Psi_{\kappa}^{(+)^{*}}(g)
$$

где

$$
\Psi_{\kappa}^{(+)}(f)=\int d^{2} x \Psi_{\kappa}^{(+)}(x) f(x)
$$

- бустова мода, сглаженная гладкой функцией $f(x) \in D\left(\mathbb{R}^{2}\right)$ с компактным носителем. Сглаженная по $x$ бустова мода (А.2) остается обобщенной функцией по переменной $\kappa$. Это означает, что $\Psi_{\kappa}^{(+)}(f)$ является линейным непрерывным функционалом на пространстве $D\left(\mathbb{R}^{1}\right)$ гладких функций с компактным носителем $\varphi(\kappa) \in$ $D\left(\mathbb{R}^{1}\right), \Psi_{\kappa}^{(+)}(f): \varphi \mapsto\left(\Psi_{\kappa}^{(+)}(f), \varphi\right)$. Тогда $\Psi_{\kappa}(f) \in D^{\prime}\left(\mathbb{R}^{1}\right)$, где $D^{\prime}\left(\mathbb{R}^{1}\right)$ - пространство обобщенных функций, дуальное к топологическому пространству $D\left(\mathbb{R}^{1}\right)$.

Можно показать, что любая бустова мода, сглаженная произвольной функцией $f(x) \in D\left(\mathbb{R}^{2}\right)$, является гладкой функцией от $\kappa$ и убывает быстрее любой степени $|\kappa|$. Однако $D^{\prime}\left(\mathbb{R}^{1}\right)$ является полным пространством (см., например, [51]) и поэтому содержит не только гладкие функции $\Psi_{\kappa}(f)$, но также и пределы всех сходящихся последовательностей таких функций. В частности, всегда можно сконструировать последовательность сглаженных бустовых мод (А.2) $\delta_{n}$ в $D^{\prime}\left(\mathbb{R}^{1}\right)$, которая сходится к $\delta(\kappa), \lim _{n \rightarrow \infty}\left(\delta_{n}, \varphi\right)=(\delta, \varphi)$ для всех $\varphi \in D\left(\mathbb{R}^{1}\right)$. Действительно, рассмотрим последовательность колоколообразных функций $f_{\sigma}(x)$ с носителем $\sigma$ с центром в точке $x=0$, нормированных на единицу, и $\sigma \rightarrow 0$. Ясно, что $\lim _{\sigma \rightarrow 0} f_{\sigma}(x)=\delta(x)$, и поэтому

$$
\lim _{\sigma \rightarrow 0} \Psi_{\kappa}^{(+)}\left(f_{\sigma}\right)=\Psi_{\kappa}^{(+)}(0)=\frac{1}{\sqrt{2}} \delta(\kappa) \in D^{\prime}\left(\mathbb{R}^{1}\right) .
$$

Это полностью согласуется с формулой (30), которая является прямым следствием трансляционной инвариантности теории. Следует отметить, что приведенное рассуждение не предполагает, что функция $\delta(x)$, являющаяся пределом последовательности $f_{\sigma}(x)$, принадлежит $D\left(\mathbb{R}^{2}\right)$, хотя $f_{\sigma}(x) \in D\left(\mathbb{R}^{2}\right)$ для каждого $\sigma \neq 0$.

Рассмотрим другой пример, позволяющий получить явные аналитические выражения для сглаженных бустовых мод (А.2). Поскольку $\Psi_{\kappa}^{(+)}(x)$ являются обобщенными функциями медленного роста по отношению к трем переменным, они, как функции от $x$, могут быть определены на более широком, чем $D\left(\mathbb{R}^{2}\right)$, пространстве основных функций, а именно на пространстве Шварца $S\left(\mathbb{R}^{2}\right)$ бесконечно дифференцируемых функций, которые быстро убывают на бесконечности. Тогда сглаженные бустовы моды $\Psi_{\kappa}^{(+)}(f)$ являются обобщенными функциями на $S\left(\mathbb{R}^{1}\right)$ и принадлежат полному пространству обобщенных функций $S^{\prime}\left(\mathbb{R}^{1}\right)$.

Выберем основные функции $f(x) \in S\left(\mathbb{R}^{2}\right)$ в виде

$$
f_{\sigma}(x)=\frac{1}{\pi \sigma^{2}} \exp \left(-\frac{t^{2}+z^{2}}{\sigma^{2}}\right)
$$

(ср. с основными функциями $f(x)$ в приложении к работе [40]). Используя для $\Psi_{\kappa}^{(+)}(x)$ представление $(20)$ и основные функции (А.4), после интегрирования по $t$ 
и $z$ получаем для сглаженных бустовых мод (А.2)

$$
\Psi_{\kappa}\left(f_{\sigma}\right)=\frac{1}{2^{5 / 2} \pi} \int_{-\infty}^{\infty} d u \exp \left(-i \frac{\kappa}{2} u-\frac{m^{2} \sigma^{2}}{4} \operatorname{ch} u\right)=\frac{1}{2^{3 / 2} \pi} K_{i \kappa / 2}\left(\frac{m^{2} \sigma^{2}}{4}\right)
$$

(см. формулу $(3.337 .1)$ в [50]). Замечая, что $K_{i \kappa / 2}(0)=2 \pi \delta(\kappa)$ [58], мы вновь приходим к (А.3).

Рассмотрим теперь сглаженную функцию Вайтмана (30). Пусть $f(x)$ - некоторая фиксированная основная функция в $D\left(\mathbb{R}^{2}\right)$ (или $S\left(\mathbb{R}^{2}\right)$ ), а $g_{\sigma}(x)$ - члены последовательности, сходящейся к $\delta(x)$. Тогда имеем числовой ряд сглаженных функций Вайтмана

$$
\Delta^{(+)}\left(f, g_{\sigma}\right)=i \int_{-\infty}^{\infty} d \kappa \Psi_{\kappa}^{(+)}(f) \Psi_{\kappa}^{(+)^{*}}\left(g_{\sigma}\right)
$$

Благодаря трансляционной инвариантности имеем $\Delta^{(+)}\left(x^{\prime}, x^{\prime \prime}\right)=\Delta^{(+)}\left(x^{\prime}-x^{\prime \prime}\right)$. Следовательно, для последовательности $\Delta^{(+)}\left(f, g_{\sigma}\right)$ получаем представление

$$
\Delta^{(+)}\left(f, g_{\sigma}\right)=i \int d^{2} x^{\prime} \int d^{2} x^{\prime \prime} f\left(x^{\prime}\right) g_{\sigma}\left(x^{\prime \prime}\right) \Delta^{(+)}\left(x^{\prime}-x^{\prime \prime}\right)=i\left(g_{\sigma} * \Delta^{(+)}\right)(f),
$$

где

$$
\left(g_{\sigma} * \Delta^{(+)}\right)(x)=\int d^{2} y g_{\sigma}(y) \Delta^{(+)}(x-y)
$$

- свертка функции $g_{\sigma}$ с обобщенной функцией $\Delta^{(+)}$. По формуле $(2-31)$ из [46]

$$
\left(g_{\sigma} * \Delta^{(+)}\right)(f)=\Delta^{(+)}\left(\hat{g}_{\sigma} * f\right),
$$

где $\hat{g}_{\sigma}(x)=g_{\sigma}(-x)$. Далее с учетом (25) и (А.6) приходим к соотношению

$$
\int_{-\infty}^{\infty} d \kappa \Psi_{\kappa}^{(+)}(f) \Psi_{\kappa}^{(+)}{ }^{*}\left(g_{\sigma}\right)=\frac{1}{\sqrt{2}} \Psi_{0}\left(\hat{g}_{\sigma} * f\right)
$$

В пределе $\sigma \rightarrow 0$

$$
\hat{g}_{\sigma}(x)=\delta(x), \quad \Psi_{\kappa}^{(+)}(\delta(x))=\Psi_{\kappa}(0), \quad \hat{g}_{\sigma} * f=\delta * f=f
$$

и формула (А.8) превращается в тривиальное тождество

$$
\Psi_{0}(f)=\int_{-\infty}^{\infty} d \kappa \Psi_{\kappa}(f) \delta(\kappa)
$$

которое является не чем иным, как сглаженным соотношением (24).

Отсюда видно, что в точности так же, как и в случае несглаженных бустовых мод, только точка $\kappa=0$ дает вклад в интегралы в (А.8), (А.9) и поэтому не может быть удалена из спектра. Объясним этот результат подробнее. 
Процедура удаления спектральной точки $\kappa=0$, например, из интеграла

$$
\frac{1}{\sqrt{2}} \Psi_{0}(f)=\lim _{\sigma \rightarrow 0} \int_{-\infty}^{\infty} d \kappa \Psi_{\kappa}^{(+)}(f) \Psi_{\kappa}^{(+)}{ }^{*}\left(g_{\sigma}\right)
$$

состоит из удаления из промежутка интегрирования $\varepsilon$-окрестности точки $\kappa=0$, вычисления интеграла и последующего перехода к пределу ${ }^{4)} \varepsilon \rightarrow 0$ :

$$
\lim _{\varepsilon \rightarrow 0} \lim _{\sigma \rightarrow 0} I_{\varepsilon}\left(f, g_{\sigma}\right), \quad I_{\varepsilon}\left(f, g_{\sigma}\right)=\left(\int_{-\infty}^{-\varepsilon}+\int_{+\varepsilon}^{+\infty}\right) d \kappa \Psi_{\kappa}^{(+)}(f) \Psi_{\kappa}^{(+)}{ }^{*}\left(g_{\sigma}\right) .
$$

Поскольку сглаженная бустова мода $\Psi_{\kappa}^{(+)^{*}}\left(g_{\sigma}\right)$ стремится к дельта-функции Дирака $\delta(\kappa)$ при $\sigma \rightarrow 0$, интеграл (А.11) в этом пределе обращается в нуль.

Проиллюстрируем это на примере последовательности основных функций $g_{\sigma}$ вида (А.4), для которых сглаженные бустовы моды даются формулой (А.5). Для простоты предположим, что $\Psi_{\kappa}(f)$ является четной функцией $\kappa, f$ также имеет вид (А.4). В этом случае имеем

$$
I_{\varepsilon}\left(f, g_{\sigma}\right)=\frac{1}{\sqrt{2} \pi} \int_{\varepsilon}^{\infty} d \kappa \Psi_{\kappa}(f) K_{i \kappa / 2}\left(\frac{m^{2} \sigma^{2}}{4}\right) .
$$

Полагая, что $\sigma$ достаточно мало, можно воспользоваться асимптотической формулой

$$
\begin{aligned}
K_{i \kappa / 2}\left(\frac{m^{2} \sigma^{2}}{4}\right) & =\frac{1}{2}\left\{\Gamma\left(\frac{i \kappa}{2}\right) e^{i \kappa \Lambda}+\Gamma\left(-\frac{i \kappa}{2}\right) e^{-i \kappa \Lambda}\right\}+O\left(\sigma^{4}\right), \\
\Lambda & =\ln \frac{2 \sqrt{2}}{m \sigma} \gg 1 .
\end{aligned}
$$

Подынтегральное выражение в (А.12) экспоненциально быстро убывает на бесконечности и в пределе $\Lambda \rightarrow \infty$ быстро осциллирует. Пользуясь стандартным методом оценки таких интегралов, получаем, что в нашем случае интеграл определяется поведением подынтегрального выражения на нижнем пределе и может быть вычислен интегрированием по частям. Так как подынтегральное выражение имеет порядок $O\left(\kappa^{-1}\right)$ при $\kappa \rightarrow 0$, следует подойти к интегрированию по частям с осторожностью 5$)$, чтобы избежать потери точности в случае малых $\varepsilon$. Действуя таким образом, получаем

$$
I_{\varepsilon}\left(f, g_{\sigma}\right)=-\frac{\sqrt{2}}{\pi} \Psi_{0}(f) \operatorname{si}(\Lambda \varepsilon)\left[1+O\left(\max \left\{\varepsilon, \Lambda^{-1}\right\}\right)\right]
$$

где

$$
\operatorname{si}(\zeta)=-\int_{\zeta}^{\infty} \frac{\sin \kappa}{\kappa} d \kappa
$$

- интегральный синус. Заметим, что на этом шаге все еще возможно избежать выкалывания точки $\kappa=0$, осуществив сначала переход к пределу $\varepsilon \rightarrow 0$. Учитывая,

\footnotetext{
4) Во избежание недоразумений отметим, что противоположный порядок пределов $\lim _{\sigma \rightarrow 0} \lim _{\varepsilon \rightarrow 0} I_{\varepsilon}\left(f, g_{\sigma}\right)$ может использоваться только в том случае, если результат не зависит от порядка перехода к пределам, просто потому, что $\lim _{\varepsilon \rightarrow 0} I_{\varepsilon}\left(f, g_{\sigma}\right)$, очевидно, восстанавливает вклад точки $\kappa=0$, и поэтому эта точка перестает быть удаленной.

5) Альтернативным способом вычисления могут быть использование регуляризации $e^{ \pm i \kappa \Lambda} \rightarrow$ $e^{ \pm i \kappa(\Lambda \pm i 0)}$ и разложение оставшейся части подынтегрального выражения в ряд Лорана при $\kappa=0$.
} 
что $\operatorname{si}(0)=-\pi / 2$, в этом случае возвращаемся к соотношению (А.10). С другой стороны, в случае исключения этой точки, как обсуждалось выше, следует сначала перейти к пределу $\sigma \rightarrow 0$. Поскольку $\operatorname{si}(\zeta)=-\cos \zeta / \zeta+O\left(\zeta^{-2}\right)$, получаем

$$
I_{\varepsilon}\left(f, g_{\sigma}\right)=\frac{\sqrt{2}}{\pi} \Psi_{0}(f) \frac{\cos \Lambda \varepsilon}{\Lambda \varepsilon}[1+O(\varepsilon)], \quad \Lambda^{-1} \ll \varepsilon \ll 1 .
$$

Отсюда очевидно, что для любого достаточно малого $\delta>0$ мы имеем $\left|I_{\varepsilon}\left(f, g_{\sigma}\right)\right|<\delta$ для всех $\sigma<\sigma_{0}(\delta, \varepsilon)=(2 \sqrt{2} / m) \exp \left[-2 \sqrt{2}\left|\Psi_{0}(f)\right| / \pi \varepsilon \delta\right]$. Это и означает, что

$$
\lim _{\varepsilon \rightarrow 0} \lim _{\sigma \rightarrow 0} I_{\varepsilon}\left(f, g_{\sigma}\right)=0 \text {. }
$$

Благодарности. Работа поддержана РФФИ (грант № 13-02-00372) и Программой государственной поддержки молодых российских ученых и государственной поддержки ведущих научных школ РФ (грант НШ-4829.2014.2).

\section{Список литературы}

[1] W. G. Unruh, Phys. Rev. D, 14:4 (1976), 870-892.

[2] P. C. W. Davies, J. Phys. A, 8:4 (1975), 609-616.

[3] В. А. Белинский, Б. М. Карнаков, В. Д. Мур, Н. Б. Нарожный, Писъма в ЖЭТФ, 65 (1997), 861-866.

[4] A. M. Fedotov, V. D. Mur, N. B. Narozhny, V.A. Belinskii, B. M. Karnakov, Phys. Lett. A, 254:3-4 (1999), 126-132, arXiv: hep-th/9902091.

[5] N. Narozhny, A. Fedotov, B. Karnakov, V. Mur, V. Belinskii, Ann. Phys. (8), 9:3 (2000), 199-206.

[6] N. B. Narozhny, A. M. Fedotov, B. M. Karnakov, V.D. Mur, V. A. Belinskii, Phys. Rev. D, 65:2 (2001), 025004, 23 pp., arXiv: hep-th/9906181.

[7] П. К. Силаев, О. А. Хрусталев, ТМФ, 91:2 (1992), 217-233.

[8] A. Arageorgis, J. Earman, L. Ruetsche, Philos. Sci., 70:1 (2003), 164-202.

[9] P. Nicolini, M. Rinaldi, Phys. Lett. B, 695 (2011), 303, arXiv: 0910.2860.

[10] D. Colosi, D. Rätzel, SIGMA, 9 (2013), 019, 22 pp.

[11] P. Chen, T. Tajima, Phys. Rev. Lett., 83:2 (1999), 256-259.

[12] R. Schützhold, G. Schaller, D. Habs, Phys. Rev. Lett., 97:12 (2006), 121302, 4 pp., arXiv: quant-ph/0604065; 100:9 (2008), 091301, 4 pp., arXiv: 0705.4385.

[13] N. Obadia, Phys. Rev. D, 76:4 (2007), 045013, 12 pp., arXiv: gr-qc/0702041.

[14] I. Fuentes-Schuller, R. B. Mann, Phys. Rev. Lett., 95:12 (2005), 120404, 4 pp., arXiv: quant-ph/0410172.

[15] P. M. Alsing, I. Fuentes-Schuller, R. B. Mann, T. E. Tessier, Phys. Rev. A, 74:3 (2006), 032326, 15 pp., arXiv: quant-ph/0603269.

[16] G. Adesso, I. Fuentes-Schuller, M. Ericsson, Phys. Rev. A, 76:6 (2007), 062112, 19 pp., arXiv: quant-ph/0701074.

[17] K. Brádler, Phys. Rev. A, 75:2 (2007), 022311, 5 pp.

[18] M. Han, S. J. Olson, J. P. Dowling, Phys. Rev. A, 78:2 (2008), 022302, 5 pp., arXiv: 0705.1350.

[19] I. Peña, D. Sudarsky, Found. Phys., 44:6 (2014), 689-708, arXiv: 1306.6621.

[20] S. De Bièvre, M. Merkli, Class. Quant. Grav., 23:22 (2006), 6525-6541.

[21] S. Takagi, Prog. Theor. Phys., 88 (1986), 1-142.

[22] H. C. Rosu, Internat. J. Modern Phys. D, 3:3 (1994), 545-548, arXiv: gr-qc/9605032.

[23] А. И. Никишов, В. И. Ритус, ЖЭТФ, 94:1(7) (1988), 31-47. 
[24] В. Д. Мур, Б. М. Карнаков, В. С. Попов, ЖЭЭФ, 114:3 (1998), 798-820.

[25] K.-P. Marzlin, J. Audretsch, Phys. Rev. D, 57:2 (1998), 1045-1051, arXiv: gr-qc/9707058.

[26] A. M. Fedotov, N. B. Narozhny, V.D. Mur, V. A. Belinskii, Phys. Lett. A, 305:5 (2002), 211-217, arXiv: hep-th/0208061.

[27] Н.Н.Боголюбов, Д. В. Ширков, Введение в теорию квантованных полей, Наука, М., 1976.

[28] E. L. Hill, Rev. Modern Phys., 23:3 (1951), 253-260.

[29] А. И. Ахиезер, В.Б. Берестецкий, Квантовая электродинамика, Наука, М., 1981.

[30] А. И. Никишов, ЖЭТФ, $\mathbf{5 7 : 4 ~ ( 1 9 6 9 ) , ~ 6 6 0 - 6 6 2 . ~}$

[31] Н. Б. Нарожный, А.И. Никишов, ТМФ, 26:1 (1976), 16-34; “Частица в постоянном электрическом поле и распространяющей вдоль него плоской электромагнитной волне", Проблемы квантовой электродинамики интенсивного поля, Труды ФИАН, 168, ред. под ред. В. Л. Гинзбурга, Наука, М., 1986, 175-199.

[32] D. M. Volkov, Z. Phys., 94:3-4 (1935), 250-260.

[33] P. J. Redmond, J. Math. Phys., 6:7 (1965), 1163-1169.

[34] D. Grumiller, W. Kummer, D. V. Vassilevich, Phys. Rep., 369:4 (2002), 327-430, arXiv: hep-th/0204253.

[35] S. V. Christensen, S. A. Fulling, Phys. Rev. D, 15:8 (1977), 2088-2104.

[36] U. Gerlach, Phys. Rev. D, 38:2 (1988), 514-521, arXiv: gr-qc/9910097.

[37] P. Longhi, R. Soldati, Phys. Rev. D, 83:10 (2011), 107701, 4 pp., arXiv: 1101.5976.

[38] P. Longhi, R. Soldati, Intern. J. Modern Phys. A, 28:21 (2013), 1350109, 25 pp., arXiv: 1210.7378 .

[39] S. A. Fulling, W. G. Unruh, Phys. Rev. D, 70:4 (2004), 048701, 4 pp.

[40] N. B. Narozhny, A. M. Fedotov, B. M. Karnakov, V. D. Mur, V. A. Belinskii, Phys. Rev. D, 70:4 (2004), 048702, 6 pp.

[41] А. М. Федотов, Н. Б. Нарожный, В.Д. Мур, Е.Г. Гельфер, Писъма в ЖЭТФ, 89:8 (2009), 449-453.

[42] M. Soffel, B. Müller, W. Greiner, Phys. Rev. D, 22:8 (1980), 1935-1937.

[43] W. Greiner, B. Müller, J. Rafelski, Quantum Electrodynamics of Strong Fields, Springer, Heidelberg, 1985.

[44] D. McMahon, P. M. Alsing, P. Embid, The Dirac equation in Rindler space: A pedagogical introduction, arXiv: gr-qc/0601010.

[45] Р. Йост, Общая теория квантованных полей, Мир, М., 1967.

[46] Р. Ф. Стритер, А. С. Вайтман, РСТ, спин и статистика и все такое, Наука, М., 1966.

[47] Н. Н. Боголюбов, А.А. Логунов, И.Т. Тодоров, Основы аксиоматического подхода в квантовой теории поля, Наука, М., 1969.

[48] А.С. Вайтман, Проблемы в релятивистской динамике квантованных полей, Наука, M., 1968.

[49] L. C. B. Crispino, A. Higuchi, G. E. A. Matsas, Rev. Modern Phys., 80:3 (2008), 787-838, arXiv: 0710.5373.

[50] И.С. Градштейн, И.М. Рыжик, Таблицы интегралов, сумм, рядов и произведений, Физматлит, М., 1971.

[51] И. М. Гельфанд, Г. Е. Шилов, Обобщенные функиии, т. 1: Обобщенные функиии и действия над ними, Физматлит, М., 1959.

[52] V.A. Fock, Z. Phys., 57:3-4 (1929), 261-277.

[53] Р. Пэли, Н. Винер, Преобразование Фуръе в комплексной области, Наука, М., 1964.

[54] Н. В. Красников, В. А. Матвеев, В. А. Рубаков, А. Н. Тавхелидзе, В. Ф. Токарев, ТМФ, 45:3 (1980), 313-328.

[55] C. Gabriel, P. Spindel, Ann. Phys., 284:2 (2000), 263-335, arXiv: gr-qc/9912016. 
[56] N. B. Narozhny, V. D. Mur, A. M. Fedotov, Phys. Lett. A, 315:3-4 (2003), 169-174, arXiv: hep-th/0304010.

[57] Е. Г. Гельфер, В. Д. Мур, Н. Б. Нарожный, А. М. Федотов, ЖЭТФ, 140:6, 1073-1087.

[58] Д. В. Львов, А. С. Шелепин, Л. А. Шелепин, ЯФ, 57:6 (1994), 1147-1152.

[59] R. Haag, Local Quantum Physics. Fields, Particles, Algebras, Springer, New York, 1992.

[60] Н. Биррелл, П. Дэвис, Квантовые поля в искривленном пространстве-времени, Мир, M., 1984.

[61] A.S. Eddington, The Nature of the Physical World, Cambridge Univ. Press, Cambridge, 1928.

Поступила в редакцию 2.04.2014 